\title{
Article
}

\section{Generic Model Control Applied to E. coli BL21(DE3) Fed-Batch Cultures}

\author{
${\text { Merouane Abadli }{ }^{1,2} \text { (D), Laurent Dewasme }}^{2}$ (D), Sihem Tebbani ${ }^{1, *}$ (D), Didier Dumur ${ }^{1}$ (D) and \\ Alain Vande Wouwer ${ }^{2, *}$ (D) \\ 1 Université Paris-Saclay, CNRS, CentraleSupélec, Laboratoire des Signaux et Systèmes, 3 Rue Joliot-Curie, \\ 91192 Gif sur Yvette, France; merouane.abadli@centralesupelec.fr (M.A.); \\ didier.dumur@centralesupelec.fr (D.D.) \\ 2 Systems, Estimation, Control and Optimization (SECO), University of Mons, 31, Boulevard Dolez, \\ 7000 Mons, Belgium; laurent.dewasme@umons.ac.be \\ * Correspondence: sihem.tebbani@centralesupelec.fr (S.T.); alain.vandewouwer@umons.ac.be (A.V.W.)
}

Received: 13 May 2020; Accepted: 28 June 2020; Published: 1 July 2020

\begin{abstract}
This work proposes a Generic Model Control (GMC) strategy to regulate biomass growth in fed-batch cultures of Escherichia coli BL21(DE3). The control law is established using a previously validated mechanistic model based on the overflow metabolism paradigm. A model reduction is carried out to prevent the controller from relying on kinetics, which may be uncertain. In order to limit the controller to the use of a single measurement, i.e., biomass concentration which is readily available, a Kalman filter is designed to reconstruct the nonmeasurable information from the outlet gas and the remaining stoichiometry. Several numerical simulations are presented to assess the controller robustness with respect to model uncertainty. Experimental validation of the proposed GMC strategy is achieved with a lab-scale bioreactor.
\end{abstract}

Keywords: bioprocess control; generic model control; linearizing control; biotechnology

\section{Introduction}

Industrial production of recombinant proteins is commonly achieved through high cell-density fed-batch fermentation of genetically modified strains of Escherichia coli, with glucose as the main or sole carbon source. This is due to the many biological traits of this microorganism, such as the flexible culture conditions, fast growth, the well-known physiological properties, the vast catalog of available tools for genetic and genomic engineering [1], and the high production yields attainable [2].

One of the main challenges in high-density fermentations is the presence of acetate, produced when E. coli growth is either performed under oxygen-limiting conditions or when the capacity for energy generation within the cell is exceeded due to high carbon flux into the main metabolic pathways $[3,4]$. As a result, acetate presence leads to a decrease of biomass production yields and consequently a decrease of the recombinant protein production $[5,6]$.

In order to maximize productivity and to avoid acetate accumulation throughout fermentation, it is important to maintain the substrate concentration to a certain threshold corresponding to the critical oxidation capacity [7], inducing the necessity to design a closed-loop feeding strategy. Several control strategies have been developed for similar fed-batch processes presenting overflow metabolism [8-12].

Accurate control of the substrate or acetate concentrations at low values may seem a straightforward approach since the process is close to the edge between the respiro-fermentative and respirative modes, where the majority of the available substrate is dedicated to biomass production. Some applications 
of this approach can be found in [13-16]. However, the lack of reliable online monitoring of acetate and glucose at low concentrations is a major hurdle for the experimental application of these methods. Additionally, the nonlinearity exhibited by the process and the need for an accurate determination of the stoichiometry and the kinetics are drawbacks in the application of model-based control strategies. Therefore, an adaptive control scheme with minimum reliance on stoichiometry and kinetics and considering online measurements of substrate and acetate can be an attractive alternative.

Generic Model Control (GMC) was developed by Lee and Sullivan [17] and is an adaptive control strategy based on feedback linearization, embedding the process nonlinearities in the design of the control law. GMC was used in various process control applications, among which we can cite, for instance [18], where GMC ensured tracking of the foreign protein level reference trajectory in E. coli fermentations [19], where it was applied to anaerobic digestion; and [20], where GMC was used to control dual product composition in an industrial high-purity distillation column. More specifically, in relation to the present study, the GMC strategy was applied in [21] to control the specific biomass growth rate in recombinant $E$. coli fed-batch cultures to an experimentally defined specific growth rate reference $\mu_{r e f}(t)$. Model simplification was carried out during the control design. In the same spirit, a control strategy was developed in [22] and applied in [23], based on an adaptive linearizing control law, regulating the acetate concentration with the dilution rate as a manipulated variable.

Taking advantage of the online measurement of the biomass concentration, the objective of the present work is to develop and implement a GMC algorithm controlling biomass productivity during the fed-batch fermentation of recombinant $E$. coli. In this control strategy, a predefined biomass trajectory corresponding to a limited acetate production is imposed by the controller. The advantages of this approach are the inclusion of the process nonlinear model in the control design and the compensation of the model inaccuracies by online adaptation using a parameter estimator. In addition, the online integration of the process model (i.e., the numerical solution of the mass balance differential equations) is not required, which leads to an easy experimental implementation.

A model order reduction is applied to avoid reliance on the specific growth rates, to reduce the computational cost, and to ensure that the process converges to the appropriate operating point.

This paper is organized as follows: In Section 2, the mechanistic model describing the growth of E. coli is detailed. The generic model control strategy is presented and applied to the E. coli model in Section 3, introducing a model order reduction to avoid the use of the kinetics in the control design. In Section 4, a Kalman filter is developed to estimate the uncertain part of the control law. The performance and robustness of the control strategy are tested in numerical simulations in Section 5. Section 6 presents the materials and methods, and experimental results are proposed and discussed in Section 7. Conclusions and perspectives end this study in Section 8.

\section{E. coli Nonlinear Dynamic Model}

This section presents the macroscopic model of E. coli cultures in fed-batch mode. The different variables, parameters, and abbreviations used in this section are described in Table 1.

The model describing biomass $(X)$ growth and acetate $(A)$ formation on glucose as the main substrate $(S)$ in fed-batch mode is based on the following biochemical reaction scheme [14]:

$$
\begin{aligned}
& S+k_{\mathrm{O} 1} \mathrm{O} \stackrel{\varphi_{1}}{\longrightarrow} k_{X 1} X+k_{\mathrm{C} 1} \mathrm{C} \\
& S+k_{\mathrm{O} 2} \mathrm{O} \stackrel{\varphi_{2}}{\longrightarrow} k_{X 2} X+k_{A 2} A+k_{\mathrm{C} 2} \mathrm{C} \\
& A+k_{\mathrm{O} 3} \mathrm{O} \stackrel{\varphi_{3}}{\longrightarrow} k_{\mathrm{X} 3} \mathrm{X}+k_{\mathrm{C} 3} \mathrm{C}
\end{aligned}
$$


where $k_{\xi i}(i=1,2,3)$ are the stoichiometric coefficients and $\varphi_{j}\left(\varphi_{j}=\mu_{j} X\right)$ are the reaction rates [22], modelled with Monod type kinetics [24]:

$$
\begin{aligned}
& \mu_{1}=\min \left(q_{s}, q_{s_{c r i t}}\right) \\
& \mu_{2}=\max \left(0, q_{s}-q_{s_{c r i t}}\right) \\
& \mu_{3}=\min \left(0, q_{A C}\right)
\end{aligned}
$$

\begin{tabular}{|c|c|c|}
\hline Variable & Description & Unit \\
\hline$x$ & biomass concentration & $\mathrm{gL}^{-1}$ \\
\hline S & glucose concentration & $\mathrm{gL}^{-1}$ \\
\hline A & acetate concentration & $\mathrm{gL}^{-1}$ \\
\hline $\mathrm{O}$ & dissolved oxygen concentration & $\mathrm{gL}^{-1}$ \\
\hline C & dissolved carbon dioxide concentration & $\mathrm{gL}^{-1}$ \\
\hline $\mathrm{V}$ & effective medium volume & $\mathrm{L}$ \\
\hline$\varphi_{1}$ & glucose oxidation reaction rate & $\mathrm{gL}^{-1} \mathrm{~h}^{-1}$ \\
\hline$\varphi_{2}$ & glucose overflow reaction rate & $\mathrm{gL}^{-1} \mathrm{~h}^{-1}$ \\
\hline$\varphi_{3}$ & acetate oxidation reaction rate & $\mathrm{gL}^{-1} \mathrm{~h}^{-1}$ \\
\hline$k_{\xi_{\{1,2,3\}}}$ & yield coefficients & $\mathrm{g} \mathrm{g}^{-1}$ \\
\hline$K_{S}$ & Monod half-saturation constant of glucose consumption & $\mathrm{gL}^{-1}$ \\
\hline$K_{A}$ & Monod half-saturation constant of acetate consumption & $\mathrm{gL}^{-1}$ \\
\hline$k_{O S}$ & yield coefficient between oxygen and substrate consumption & $\mathrm{gL}^{-1} \mathrm{~h}^{-1}$ \\
\hline$K_{O A}$ & yield coefficient between oxygen and acetate consumption & $\mathrm{gL}^{-1} \mathrm{~h}^{-1}$ \\
\hline$K_{i A}$ & constant of growth inhibition by acetate & $\mathrm{gL}^{-1} \mathrm{~h}^{-1}$ \\
\hline$q_{s}$ & substrate consumption rate & $\mathrm{gL}^{-1} \mathrm{~h}^{-1}$ \\
\hline$q_{s_{\text {crit }}}$ & critical substrate consumption rate & $\mathrm{gL}^{-1} \mathrm{~h}^{-1}$ \\
\hline$q_{A C}$ & acetate consumption rate & $\mathrm{gL}^{-1} \mathrm{~h}^{-1}$ \\
\hline$F_{\text {in }}$ & feed flow rate & $\mathrm{Lh}^{-1}$ \\
\hline$D$ & dilution rate $\left(D=F_{\text {in }} / V\right)$ & $\mathrm{h}^{-1}$ \\
\hline$S_{\text {in }}$ & substrate concentration in the feed medium & $\mathrm{gL}^{-1}$ \\
\hline OTR & oxygen transfer rate from the gas phase to the liquid phase & $\mathrm{gL}^{-1} \mathrm{~h}^{-1}$ \\
\hline CTR & carbon dioxide transfer rate from the liquid to the gas phase & $\mathrm{gL}^{-1} \mathrm{~h}^{-1}$ \\
\hline$k_{L} a_{O}$ & volumetric transfer coefficient of oxygen & $\mathrm{h}^{-1}$ \\
\hline$k_{\mathrm{L}} a_{\mathrm{CO}_{2}}$ & volumetric transfer coefficient of carbon dioxide & $\mathrm{h}^{-1}$ \\
\hline$O_{\text {sat }}$ & dissolved oxygen concentration at saturation & $\mathrm{gL}^{-1}$ \\
\hline $\mathrm{CO}_{2 \text { sat }}$ & dissolved carbon dioxide concentration at saturation & $\mathrm{gL}^{-1}$ \\
\hline
\end{tabular}

Table 1. Variables and abbreviations.

The consumption rates are defined by

$$
\begin{aligned}
q_{s} & =q_{s_{\max }} \frac{S}{K_{s}+S} \\
q_{s_{\text {crit }}} & =\frac{q_{O_{\max }}}{k_{O S}} \frac{K_{i A}}{K_{i A}+A} \\
q_{A C} & =\frac{k_{O S}\left(q_{s_{\text {crit }}}-q_{s}\right)}{k_{O A}} \frac{A}{K_{A}+A}
\end{aligned}
$$

where $q_{s}$ and $q_{A C}$ denote the substrate and acetate consumption rates, respectively; $q_{s_{\max }}$ and $q_{O_{\max }}$ are the maximal consumption rates for substrate and dissolved oxygen, respectively; and $q_{s_{c r i t}}$ represents the critical substrate consumption rate. 
The kinetic model (2) is based on the bottleneck assumption of Sonnleitner and Käppeli [25]. According to the substrate concentration level, two different operating modes can be distinguished. A substrate concentration higher than the critical value corresponding to the available oxidative capacity $\left(S>S_{c r i t}\right)$ leads to acetate formation through the fermentative metabolic pathway (reactions (1a) and (1b)). The system is said to be in respiro-fermentative mode. On the other hand, a low substrate concentration $\left(S<S_{\text {crit }}\right)$ leads to substrate and acetate (if present) oxidation, and the system is said to be in respirative mode (reactions (1a) and (1c)).

Applying mass balances to the reaction scheme (1), we obtain [24] the following:

$$
\begin{aligned}
\dot{X} & =\left(k_{X 1} \mu_{1}+k_{X 2} \mu_{2}+k_{X 3} \mu_{3}\right) X-D X \\
\dot{S} & =-\left(\mu_{1}+\mu_{2}\right) X-D\left(S-S_{i n}\right) \\
\dot{A} & =\left(k_{A 2} \mu_{2}-\mu_{3}\right) X-D A \\
\dot{O} & =-\left(k_{O 1} \mu_{1}+k_{O 2} \mu_{2}+k_{O 3} \mu_{3}\right) X-D O+O T R \\
\dot{C} & =\left(k_{C 1} \mu_{1}+k_{C 2} \mu_{2}+k_{C 3} \mu_{3}\right) X-D C-C T R \\
\dot{V} & =F_{i n}
\end{aligned}
$$

where $D$ is the dilution rate defined by $D=\frac{F_{i n}}{V}$. In the following, we consider the feed-rate $F_{\text {in }}$ as the manipulated variable.

$O T R$ and CTR can be expressed with the following basic expressions [26]:

$$
\begin{aligned}
& \text { OTR }=k_{L} a_{O}\left(O_{\text {sat }}-O\right) \\
& \text { CTR }=k_{L} a_{\mathrm{CO}_{2}}\left(\mathrm{CO}_{2}-\mathrm{CO}_{2 \mathrm{sat}}\right)
\end{aligned}
$$

\section{Generic Model Control}

Generic Model Control (GMC) is based on the input-output linearization of the nonlinear dynamics of the system of (4). The main objective is to track a desired output nominal trajectory [27]. Consider a process described as follows:

$$
\begin{aligned}
& \dot{x}=f(x)+g(x) u \\
& y=h(x)
\end{aligned}
$$

where $x \in \mathbb{R}^{n}$ is the state vector, $u \in \mathbb{R}$ is the manipulated input, and $y \in \mathbb{R}$ is the system output. $f: \mathbb{R}^{n} \rightarrow \mathbb{R}^{n} g: \mathbb{R}^{n} \rightarrow \mathbb{R}^{n}$ are nonlinear functions of the states $x$, and $h: \mathbb{R}^{n} \rightarrow \mathbb{R}$ is the output map. From Equation (8), the output dynamics are given by the following:

$$
\dot{y}=\frac{\partial h}{\partial x}[f(x)+g(x) u]=L_{f} h(x)+L_{g} h(x) u
$$

where $L_{f} h(x)=\frac{\partial h}{\partial x} f(x)$ is the lie derivative of $h$ along $f$.

The idea is to force $\dot{y}$ to follow a prescribed dynamics defined by a specification input $\hat{u}$, i.e., $\dot{y}=\hat{u}$. If $L_{\mathrm{g}} h(\mathrm{x}) \neq 0$ (i.e., the system is of relative degree 1), the control input is then given by the following:

$$
u=\frac{1}{L_{\mathrm{g}} h}\left(-L_{f} h+\hat{u}\right)
$$


The specification signal $\hat{u}$ can be defined using a proportional integral controller in the following form:

$$
\dot{y}=\hat{u}=G_{1}\left(y_{r e f}-y\right)+G_{2} \int_{0}^{t}\left(y_{r e f}-y\right) \partial \tau
$$

where $y_{r e f}$ is the reference output and where $G_{1}$ and $G_{2}$ are tuning gains, for which the values are chosen according to the desired dynamic behavior.

From Equation (11), it is obvious that the resulting closed-loop transfer function (Figure 1) is given by the following:

$$
\frac{Y(s)}{Y_{r e f}(s)}=\frac{s\left(G_{1}+G_{2}\right)}{s^{2}+G_{1} s+G_{2}}
$$

where $Y(s)$ and $Y_{r e f}(s)$ are respectively the Laplace transforms of $y$ and $y_{r e f} . s$ is the Laplace variable.

Although the controlled system response is different from the classic second-order system due to nonlinearity, similar plots of the closed-loop response for different values of $\xi$ and $\omega_{0}$ can be obtained. An example is given in Figure A1 in Appendix B with multiple responses for the studied process.

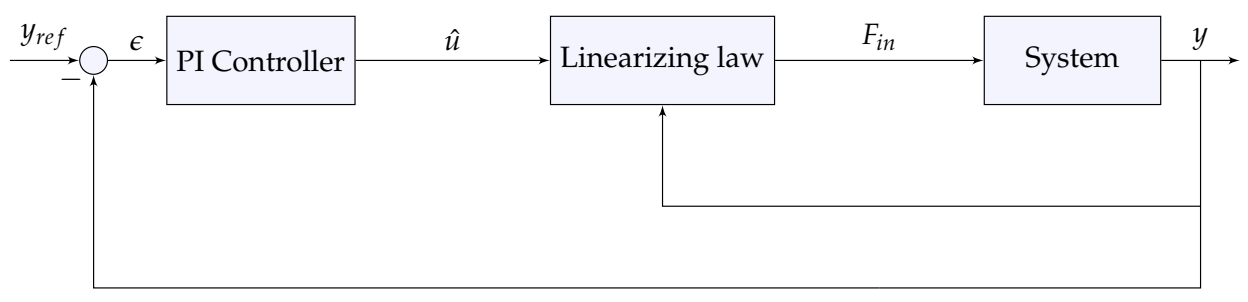

Figure 1. Generic Model Control (GMC) structure.

$G_{1}$ and $G_{2}$ can be tuned so as to confer the desired damping ratio $\xi$ and a natural frequency $\omega_{0}$ in the following steps:

- Choose $\xi$ according to the desired response shape from Figure A1.

- Choose an appropriate rise time $t_{r}$ and the corresponding $\omega_{0}=\frac{3}{t_{r}}$.

- Calculate $G_{1}$ and $G_{2}$ using the following equations:

$$
\left\{\begin{array}{l}
G_{1}=2 \xi \omega_{0} \\
G_{2}=\omega_{0}^{2}
\end{array}\right.
$$

The nonlinear closed-loop stability and the performance analysis of the GMC are detailed in [28]. Nominal stability is ensured for any positive values of $G_{1}$ and $G_{2}$. The proof is based on finding a strict Lyapunov function for the nominal process and on applying a perturbation theorem. Another stability proof for a similar control structure with kinetic parameter estimation is given in [11].

\subsection{GMC Applied to E. coli Cultures}

The specific control objective in the fed-batch cultures of E. coli is to favor biomass production and to reach high cell densities while avoiding acetate accumulation and its growth inhibiting effect. This is achieved by determining a feed manipulation strategy tracking the imposed operating conditions, ideally at the boundary between the respiro-fermentative and the respirative modes, where the substrate concentration is neither limiting nor in excess $\left(S=S_{c r i t}\right.$ and $\left.q_{s}=q_{s c r i t}\right)$.

The classical control approaches consider either controlling the substrate concentration accurately at the critical level (optimal) or controlling the byproduct (acetate) concentration at a low value (suboptimal). The main obstacles to implement these two approaches are the metabolic switches between modes in the neighborhood of the critical substrate concentration and the sensitivity limitations of the available 
online acetate and substrate measurement devices. Indeed, the glucose critical level is very low $\left(O\left(10^{-2}\right)\right.$ $\left.\mathrm{gL}^{-1}\right)$, and the acetate concentration at this critical level is equal to zero. Therefore, soft sensors with high estimation accuracy and robustness are required.

In this work, we consider the tracking of a predetermined suboptimal biomass trajectory. This approach has the merit of being pragmatic and inexpensive, as only an online biomass measurement is required using a turbidity probe, with an advantage being the low measurement noise level of such probes.

\subsubsection{GMC Design Using the Full-Order Model}

Considering biomass as the sole online measurement $(y=X)$ and using the model Equation (4a) and Equation (9), we obtain the following control law:

$$
F_{i n}=\frac{V}{X}\left(\left(k_{X 1} \mu_{1}+k_{X 2} \mu_{2}+k_{X 3} \mu_{3}\right) X-\hat{F}\right)
$$

provided that $X \neq 0$. In this expression, $\hat{F}$ is given by the following:

$$
\hat{F}=G_{1}\left(X_{r e f}-X\right)+G_{2} \int_{0}^{t}\left(X_{r e f}-X\right) \partial \tau
$$

The main limitation of the straightforward application of GMC is the difficulty to accurately determine the specific growth rates, since the kinetics are based on the overflow metabolism paradigm and are represented by metabolic switches. Moreover, an imposed biomass trajectory could possibly lead to high flow-rates.

The feeding trajectory $F_{i n_{r e f}}$ corresponding to a set biomass trajectory $X_{s e t}$ is determined hereafter. The biomass differential equation in respiro-fermentative mode is given by the following:

$$
\dot{X}=\mu_{X} X-\frac{F_{i n}}{V} X
$$

where

$$
\mu_{X}=\left(k_{X 1}-k_{X 2}\right) q_{s_{c r i t}}+k_{X 2} q_{s}
$$

If the imposed biomass trajectory is exponential with a constant rate $\mu_{\text {set }}$, the following feeding profile is obtained:

$$
F_{\text {in } n_{\text {ref }}}=\left.\left(\mu_{X}-\mu_{\text {set }}\right) V\right|_{X=X_{\text {set }}}
$$

The substrate dynamics is assumed to be fast and, therefore, in quasy-steady state $\left(S=S_{\text {set }}, \dot{S}=0\right)$, implying the following:

$$
\dot{S}=\mu_{S} X-\frac{F_{\text {in }}}{V}\left(S-S_{\text {in }}\right)=0
$$

where

$$
\mu_{S}=\left.q_{s_{\max }} \frac{S}{K_{S}+S}\right|_{S=S_{\text {set }}}
$$

which leads to the following feed-rate profile:

$$
F_{i n_{r e f}}=\left.\frac{\mu_{S}}{\left(S_{i n}-S\right)} X V\right|_{S=S_{\text {set }}}
$$


From Equations (18) and (21), the reference feeding trajectory depends on the couple $\left(X_{\text {set }}, S_{\text {set }}\right)$ :

$$
\left.\frac{\mu_{S}}{\left(S_{\text {in }}-S\right)}\right|_{X_{\text {set }}, S_{\text {set }}}=\left.\left(\frac{\mu_{X}-\mu_{\text {set }}}{X}\right)\right|_{S_{\text {set }}, X_{\text {set }}}
$$

For each value of $X_{\text {set }}$, two possible solutions for $S_{\text {set }}$ are obtained:

$$
S_{\text {set }}=\left(\alpha_{1}+\alpha_{2} X_{\text {set }} \pm \sqrt{\alpha_{3}+\alpha_{4} X_{\text {set }}+\alpha_{5} X_{\text {set }}^{2}}\right) / \alpha_{6}
$$

The expressions of the $\alpha_{j}$ coefficients are given in appendix A. A numerical example with $\mu_{\text {set }}=$ $0.18 \mathrm{~h}^{-1}$ and $X_{\text {set }}=10 \mathrm{gL}^{-1}$ is presented in Table 2. The first solution $S_{\text {set }_{1}}$ is a low value of $S$, while the second solution $S_{\text {set }_{2}}$ is rejected because it corresponds to a high value and physically non-achievable operating condition.

Table 2. $\alpha_{j}$ values and $S_{\text {set }}$ solutions for $\mu_{\text {set }}=0.18 \mathrm{~h}^{-1}$ and $X_{\text {set }}=10 \mathrm{gL}^{-1}$.

\begin{tabular}{ll}
\hline$\alpha_{1}=-1.5602 \times 10^{4}$ & $\alpha_{2}=164.0900$ \\
$\alpha_{3}=2.4338 \times 10^{8}$ & $\alpha_{4}=-5.1201 \times 10^{6}$ \\
$\alpha_{5}=2.6926 \times 10^{4}$ & $\alpha_{6}=-62.4058$ \\
$S_{\text {set }_{1}}=0.0294 \mathrm{gL}^{-1}$ & $S_{\text {set }_{2}}=447.4088 \mathrm{gL}^{-1}$ \\
\hline
\end{tabular}

\subsubsection{GMC Design Using a Reduced Model}

A control design based on a reduced model is developed by applying the singular perturbation technique [14]: the dynamics of substrate, oxygen, and carbon dioxide are considered much faster than the dynamics of biomass and acetate. Thus, the fast variables are considered to be in quasi steady-state and their dynamics are set to zero.

A fast-slow state partition is therefore proposed as follows:

$$
\xi_{f}=\left(\begin{array}{l}
S \\
O \\
C
\end{array}\right) \quad \xi_{s}=\left(\begin{array}{c}
X \\
A
\end{array}\right)
$$

with indices $f$ and $s$ being respectively related to fast and slow dynamics. The dynamic system for the fast variables can be written from (4a) to (4f):

$$
\underbrace{\left(\begin{array}{c}
\dot{S} \\
\dot{O} \\
\dot{C}
\end{array}\right)}_{\dot{\zeta}_{f}}=\underbrace{\left(\begin{array}{ccc}
-1 & -1 & 0 \\
-k_{O 1} & -k_{O 2} & -k_{O 3} \\
k_{C 1} & k_{C 2} & k_{C 3}
\end{array}\right)}_{K_{f}} \underbrace{\left(\begin{array}{l}
\varphi_{1} \\
\varphi_{2} \\
\varphi_{3}
\end{array}\right)}_{\varphi}-\underbrace{D\left(\begin{array}{c}
S \\
O \\
C
\end{array}\right)}_{\tilde{\zeta}_{f}}+\underbrace{\left(\begin{array}{c}
D S_{i n} \\
\text { OTR } \\
-C T R
\end{array}\right)}_{F_{f}+Q_{f}}
$$

where

- $K_{f}$ is the stoichiometric matrix.

- $\varphi$ is the reaction rate vector defined as $\varphi=\left(\begin{array}{lll}\mu_{1} X & \mu_{2} X & \mu_{3} X\end{array}\right)^{T}$

Since the dynamics of these variables $\left(\dot{\zeta}_{f}=0\right)$ as well as the dilution effects $\left(D \xi_{f}=\frac{F_{i n}}{V} \xi_{f}=0\right)$ are neglected, which are often several orders of magnitude smaller than the reaction terms, the following equation holds approximately:

$$
K_{f} \varphi+F_{f}+Q_{f}=0
$$


If $K_{f}$ is full rank, Equation (26) can be rewritten as follows:

$$
\varphi=K_{f}^{-1}\left(-Q_{f}-F_{f}\right)
$$

The state space dynamics of the reduced model considering only slow variables is given by the following:

$$
\underbrace{\left(\begin{array}{c}
\dot{X} \\
\dot{A}
\end{array}\right)}_{\dot{\xi}_{s}}=\underbrace{\left(\begin{array}{ccc}
k_{X 1} & k_{X 2} & k_{X 3} \\
0 & k_{A 1} & -1
\end{array}\right)}_{K_{S}} \underbrace{\left(\begin{array}{l}
\varphi_{1} \\
\varphi_{2} \\
\varphi_{3}
\end{array}\right)}_{\varphi}-\frac{F_{i n}}{V} \underbrace{\left(\begin{array}{c}
X \\
A
\end{array}\right)}_{\tilde{\xi}_{s}}
$$

Replacing the expression of $\varphi$ from (27) in (28) yields the following:

$$
\dot{\xi}_{s}=\bar{K}\left(-Q_{f}-F_{f}\right)-\frac{F_{i n}}{V} \xi_{s}
$$

where

$$
\bar{K}=K_{s} K_{f}^{-1}=\left(\begin{array}{lll}
k_{11}^{-} & k_{12}^{-} & k_{13}^{-} \\
k_{21}^{-} & k_{22}^{-} & k_{23}^{-}
\end{array}\right)
$$

The following equation are for the biomass:

$$
\dot{X}=-k_{11}^{-} \frac{F_{\text {in }}}{V} S_{\text {in }}-\overline{k_{12}} \text { OTR }+\overline{k_{13}^{-}} C T R-\frac{F_{\text {in }}}{V} X
$$

where the parameters $\overline{k_{11}}, \overline{k_{12}}$, and $\overline{k_{13}}$ are functions of the yield coefficients shown in Table 3 .

Table 3. Theoritical dependency of $\overline{k_{i j}}$ parameters.

\begin{tabular}{lc}
\hline Parameter & Expression \\
\hline$k_{11}^{-}$ & $\overline{1}\left(k_{\mathrm{X} 3}\left(k_{\mathrm{C} 1} k_{\mathrm{O} 2}\right)-k_{\mathrm{C} 2} k_{\mathrm{O} 1}-k_{\mathrm{X} 2}\left(k_{\mathrm{C} 1} k_{\mathrm{O} 3}-k_{\mathrm{C} 3} k_{\mathrm{O} 1}\right)+k_{\mathrm{C} 3} k_{\mathrm{O} 2}\right)+k_{\mathrm{X} 1}\left(k_{\mathrm{C} 2} k_{\mathrm{O} 3}-k_{\mathrm{C} 3} k_{\mathrm{O} 2}\right)$ \\
$\overline{k_{12}}$ & $\overline{\bar{\delta}}\left(k_{\mathrm{C} 3} k_{\mathrm{X} 1}\right)-\left(k_{\mathrm{C} 3} k_{\mathrm{X} 2}\right)-\left(k_{\mathrm{X} 3}\left(k_{\mathrm{C} 1}-k_{\mathrm{C} 2}\right)\right)$ \\
$k_{13}^{-}$ & $\left.\overline{\frac{\delta}{\delta}}\left(k_{\mathrm{O} 3} k_{\mathrm{X} 1}\right)-\left(k_{\mathrm{O} 3} k_{\mathrm{X} 2}\right)-k_{\mathrm{X} 3}\left(k_{\mathrm{O} 1} k_{\mathrm{O} 2}\right)\right)$ \\
$\bar{\delta}$ & $\left(k_{\mathrm{C} 1} k_{\mathrm{O} 3}-k_{\mathrm{C} 3} k_{\mathrm{O} 1}-k_{\mathrm{C} 2} k_{\mathrm{O} 3}+k_{\mathrm{C} 3} k_{\mathrm{O} 2}\right)$ \\
\hline
\end{tabular}

From Equations (9), (15) and (31), the following control law is obtained:

$$
F_{i n}=\frac{-k_{12}^{-} O T R+k_{13}^{-} C T R-\hat{F}}{X+k_{11}^{-} S_{i n}} V
$$

where $F_{\text {in }}$ is still given by Equation (15) and assuming that $X+k_{11}^{-} S_{i n} \neq 0$.

The advantage of the model reduction is that the desired operating condition (low substrate concentration) is directly embedded in the control algorithm.

Since OTR and CTR are not available for online measurement in our experimental setup and the biomass $X$ is the sole measured variable, a parameter estimator is developed in the next section to reconstruct the unavailable signals, also adapting the control law subject to parameter uncertainty. 


\section{Parameter Estimation}

Equation (31) for the biomass dynamics can be rewritten as follows:

$$
\dot{X}=\gamma-D\left(X+k_{11}^{-} S_{i n}\right)
$$

where $D=\frac{F_{i n}}{V}$ and $\gamma$ is the uncertain and unmeasurable time-varying parameter given by the following:

$$
\gamma=-k_{12}^{-} O T R+\overline{k_{13}} C T R
$$

Let

$$
X^{*}=X+\bar{k}_{11}^{-} S_{i n}
$$

Hence, Equation (33) can be written in a compact form:

$$
\dot{X}^{*}=\gamma-D X^{*}
$$

$\gamma$ can be estimated using a linear Kalman filter in the same way as presented in [11], where the production rate of lactic acid is estimated in continuous mode.

Two estimation approaches are presented in the following, based on the classical discrete Kalman filter which offers simplicity and easy implementation.

\subsection{Constant Evolution of $\gamma$}

First, $\gamma$ is assumed to be constant, which is described by an exogeneous system:

$$
\left\{\begin{array}{cc}
\dot{X}^{*}= & \gamma-D X^{*} \\
\dot{\gamma}= & 0
\end{array}\right.
$$

As biomass measurements are collected at discrete time (sampling time $T_{s}$ ), it can be convenient to discretize Equation (37) using, for instance, the Euler scheme:

$$
\begin{gathered}
{\left[\begin{array}{c}
\hat{X}_{k+1}^{*} \\
\hat{\gamma}_{k+1}
\end{array}\right]=\left[\begin{array}{cc}
-D_{k} T_{s}+1 & T_{s} \\
0 & 1
\end{array}\right]\left[\begin{array}{l}
\hat{X}_{k}^{*} \\
\hat{\gamma}_{k}
\end{array}\right]+\left[\begin{array}{l}
v_{1, k} \\
v_{2, k}
\end{array}\right]} \\
\hat{X}_{k}^{*}=\left[\begin{array}{ll}
1 & 0
\end{array}\right]\left[\begin{array}{l}
\hat{X}_{k}^{*} \\
\hat{\gamma}_{k}
\end{array}\right]+w_{k}
\end{gathered}
$$

where $D_{k}$ is the dilution rate at the time instant $t_{k}\left(D_{k}=\frac{F_{i n_{k}}}{V_{k}}\right)$, assumed to be piece-wise constant. $v_{k}$ and $w_{k}$ are respectively the process and measurement noises, assumed to be centered Gaussian white noises with covariance matrices $Q_{\text {const }}$ and $R_{\text {const }}$, respectively. The covariance matrix of the error on the state vector $\left[X_{k} \gamma_{k}\right]^{T}$ is denoted $P_{\text {const }}$.

\subsection{Ramp Evolution of $\gamma$}

Considering a ramp evolution of $\gamma$, Equation (37) becomes the following:

$$
\left\{\begin{array}{cc}
\dot{X}^{*}= & \gamma-D X^{*} \\
\ddot{\gamma}= & 0
\end{array}\right.
$$


As in the previous case, the model is discretized and additive noises are considered:

$$
\begin{aligned}
{\left[\begin{array}{c}
\hat{X}_{k+1}^{*} \\
\hat{\gamma}_{k+1} \\
\hat{\gamma}_{k+1}
\end{array}\right] } & =\left[\begin{array}{ccc}
-D_{k} T_{s}+1 & T_{s} & 0 \\
0 & 1 & T_{s} \\
0 & 0 & 1
\end{array}\right]\left[\begin{array}{c}
\hat{X}_{k}^{*} \\
\hat{\gamma}_{k} \\
\hat{\gamma}_{k}
\end{array}\right]+\left[\begin{array}{c}
v_{1, k} \\
v_{2, k} \\
v_{3, k}
\end{array}\right] \\
\hat{X}_{k}^{*} & =\left[\begin{array}{lll}
1 & 0 & 0
\end{array}\right]\left[\begin{array}{c}
\hat{X}_{k}^{*} \\
\hat{\gamma}_{k} \\
\hat{\gamma}_{k}
\end{array}\right]+w_{k}
\end{aligned}
$$

The covariance matrices of $v_{k}$ and $w_{k}$ as well as the error on the state vector $\left[\begin{array}{lll}X_{k} & \gamma_{k} & \dot{\gamma}_{k}\end{array}\right]^{T}$ are denoted $Q_{\text {Lin }}, R_{\text {Lin }}$, and $P_{\text {Lin }}$, respectively.

\subsection{Kalman Filtering}

A discrete Kalman filter is then used to estimate $X^{*}$ and $\gamma$ [29]. The control structure with the Kalman filter estimation is illustrated in Figure 2.

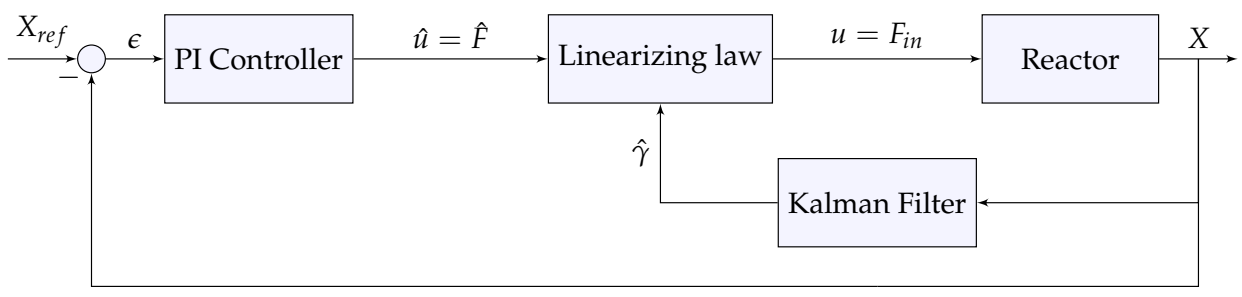

Figure 2. GMC combined with the Kalman filter.

The control law after including the estimated parameter $\hat{\gamma}$ becomes the following:

$$
F_{i n}=\frac{\hat{\gamma}-\hat{F}}{X+k_{11}^{-} S_{\text {in }}} V
$$

An anti-windup mechanism is also added to the integral term of $\hat{F}$ to avoid integration accumulation under input saturation:

$$
\hat{F}=G_{1}\left(X_{r e f}-X\right)+G_{2} \int_{0}^{t}\left[\left(X_{r e f}-X\right)+K_{\omega}\left(u_{s a t}-u\right)\right] d \tau
$$

where $K_{\omega}$ is the anti-windup gain.

As it will become apparent in the next section, both exogeneous models yield similar performances in simulations.

\section{Numerical Simulation}

The model parameters used in the simulation study are taken from [24] and are given in Table 4. The initial conditions and the different parameters of the controller and the estimator are presented in Table 5.

The imposed exponential feed rate corresponding to a constant and low subtrate concentration is given by the following:

$$
F_{i_{\text {ref }}}=\left.\frac{\mu_{S}}{\left(S_{\text {in }}-S\right)} X V\right|_{S=S_{\text {set }}}
$$


From the model equations (4) and since $\mu_{X}$ is constant, we obtain the following:

$$
\frac{d(X V)}{d t}=\mu_{X} X V
$$

Hence,

$$
X V=X_{0} V_{0} e^{\mu_{X} t}
$$

where $V_{0}$ and $X_{0}$ denote the initial volume and biomass concentrations, respectively. The following feeding profile is obtained:

$$
F_{i n_{\text {ref }}}=\frac{\mu_{S}}{\left(S_{\text {in }}-S\right)} X_{0} V_{0} e^{\mu_{X} t}
$$

In this study, the considered $F_{i n_{r e f}}$ profile given in [24] is applied:

$$
F_{i n_{r e f}}=\frac{\mu_{s e t}}{k_{X S}} \frac{X_{0} V_{0} e^{\mu_{s e t} t}}{S_{i n}}
$$

where $\mu_{\text {set }}$ is the setpoint specific growth rate and $k_{X S}$ is the yield coefficient defined as grams of produced biomass per grams of consumed substrate.

Table 4. Numerical values of the E. coli fed-batch model parameters (as in [24]).

\begin{tabular}{ccc}
\hline Parameter & Value & Unit \\
\hline$k_{X 1}$ & 0.184 & $\mathrm{~g} \mathrm{~g}^{-1}$ \\
$k_{X 2}$ & 0.2899 & $\mathrm{~g} \mathrm{~g}^{-1}$ \\
$k_{X 3}$ & 0.0412 & $\mathrm{~g} \mathrm{~g}^{-1}$ \\
$k_{A 2}$ & 0.4324 & $\mathrm{~g} \mathrm{~g}^{-1}$ \\
$q_{s_{\max }}$ & 3.2818 & $\mathrm{~h}^{-1}$ \\
$q_{o_{\max }}$ & 1.4032 & $\mathrm{~h}^{-1}$ \\
$K_{S}$ & 0.0502 & $\mathrm{gL}^{-1}$ \\
$K_{i A}$ & 2.0410 & $\mathrm{gL}^{-1}$ \\
$K_{A}$ & 0.3928 & $\mathrm{gL}^{-1}$ \\
$k_{O S} / k_{O A}$ & 1 & - \\
$k_{O 1}$ & 0.7374 & $\mathrm{~g} \mathrm{~g}^{-1}$ \\
$k_{O 2}$ & 0.3194 & $\mathrm{~g} \mathrm{~g}^{-1}$ \\
$k_{O 3}$ & 1.3418 & $\mathrm{~g} \mathrm{~g}^{-1}$ \\
$k_{\mathrm{C} 1}$ & 0.7608 & $\mathrm{~g} \mathrm{~g}^{-1}$ \\
$k_{\mathrm{C} 2}$ & 0.1055 & $\mathrm{~g} \mathrm{~g}^{-1}$ \\
$k_{C 3}$ & 0.8461 & $\mathrm{~g} \mathrm{~g}^{-1}$ \\
$S_{i n}$ & 500 & $\mathrm{gL}^{-1}$ \\
\hline
\end{tabular}

First, the Kalman filter is tested with both the constant and ramp exogeneous models for $\gamma$. Figure 3 shows the evolution of the variable $\gamma$ constructed with the computation of OTR and CTR and its estimate using both the constant and ramp exogeneous models. The estimator convergence is achieved in $20 \mathrm{mn}$ after a transient phase. Both exogeneous models yield good performance of the Kalman filter. In the following (and in the experiments), the ramp model is selected for its slightly better results, shown in Table 6.

Next, the control strategy is tested assuming that the variables are directly measurable (no Kalman filter). The biomass reference trajectory is chosen as an exponential feed rate, corresponding to a constant growth rate, an initial substrate concentration lower than the critical value $\left(S^{*}<S_{\text {crit }}\right)$, and an initial 
acetate concentration equal to zero. This operating trajectory allows the process to evolve close to the edge between the respirative and respiro-fermentative modes, where cell growth is maximized. The biomass reference trajectory should be carefully selected: $\mu_{\text {set }} \leq \mu_{X}^{\star}$, where $\mu_{X}^{\star}$ is the optimal growth rate. Simulation of biomass productivity and acetate production for different values of $\mu_{\text {set }}$ are given in Figure 4. Biomass productivity increases for increasing $\mu_{\text {set }}$ values until $\mu_{\text {set }}=0.24 \mathrm{~h}^{-1}$. For the sake of security with respect to possible model uncertainties offsetting $\mu_{X}, \mu_{\text {set }}$ will be selected in the range $[0.18,0.22]$ in order to avoid acetate accumulation.

Table 5. Control and estimation parameters.

\begin{tabular}{cc}
\hline Initial conditions & $\left(\begin{array}{c}X_{0} \\
S_{0} \\
A_{0} \\
V_{0}\end{array}\right)\left(\begin{array}{c}1.42 \mathrm{gL}^{-1} \\
0.5 \mathrm{gL}^{-1} \\
0.5 \mathrm{gL}^{-1} \\
3.15 \mathrm{~L}\end{array}\right)$ \\
\hline \multirow{4}{*}{ Control parameters } & $T_{e}=0.05 \mathrm{~h}$ \\
$G_{1}=6, G_{2}=9$ \\
$\xi=1, t_{r}=1 \mathrm{~h}$ \\
$\omega_{0}=\frac{3}{t_{r}}=3 \mathrm{rad} / \mathrm{h}$ \\
\hline \multirow{3}{*}{ Reference characteristics } & $F_{\text {in }}=\frac{\mu_{\text {set }}}{k_{X S}} \frac{V_{0} X_{0} e^{\mu_{\text {set }}(t)}}{S_{\text {in }}}$ \\
& $\mu_{\text {set }}=0.18 \mathrm{~h}^{-1}, k_{X S}=0.2$ \\
\hline \multirow{2}{*}{ Estimator covariance matrices } & $P_{\text {Lin }}=\operatorname{diag}([0.111])$ \\
& $Q_{\text {Lin }}=\operatorname{diag}([0.010 .10 .1])$ \\
& $P_{\text {Const }}=\operatorname{diag}([0.11])$ \\
& $Q_{\text {Const }}=\operatorname{diag}([0.010 .1])$ \\
& $R_{\text {Const }}=R_{\text {Lin }}=0.1$ \\
\hline
\end{tabular}

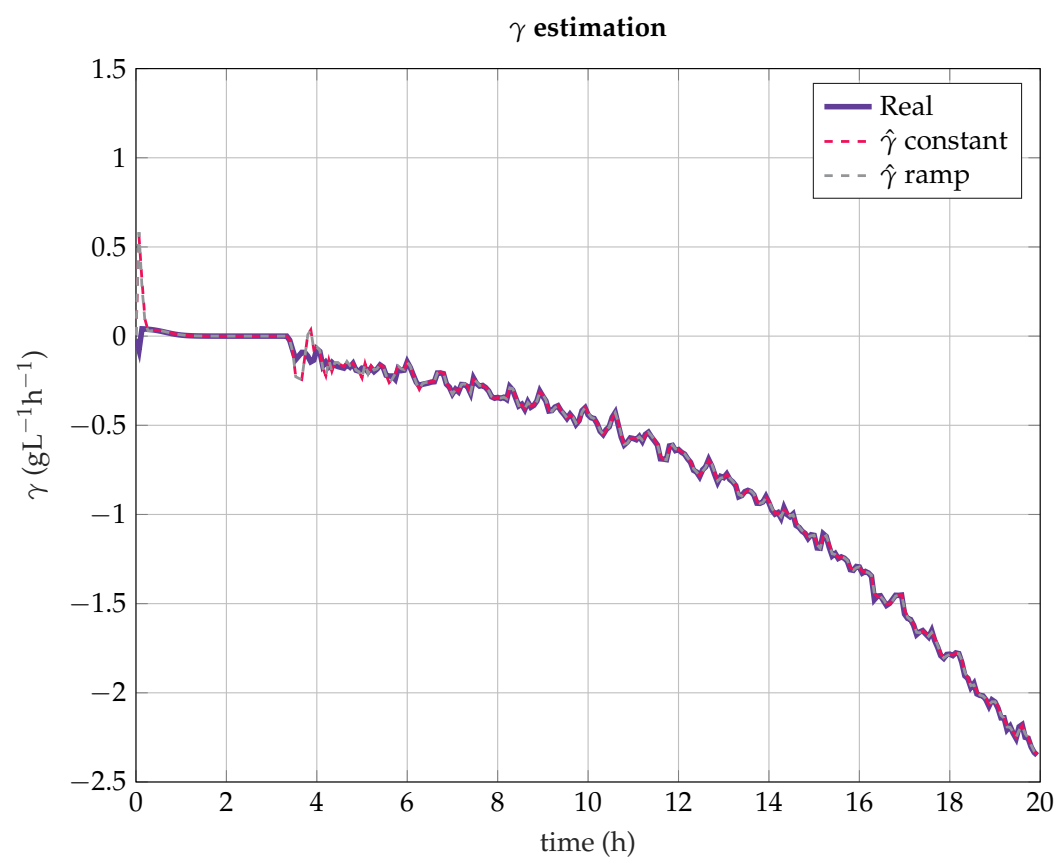

Figure 3. $\hat{\gamma}$ estimation based on biomass measurement using both constant and ramp exogeneous models.

Table 6. Root Mean Square error (RMSE) comparison between the constant and ramp exogeneous models.

\begin{tabular}{ccc}
\hline Model & Constant & Ramp \\
\hline RMSE $\left(\mathrm{gL}^{-1} \mathrm{~h}^{-1}\right)$ & 0.0872 & 0.0860 \\
\hline
\end{tabular}


Figures 5-7 show the simulation results of the GMC strategy based on the nominal and reduced models. The initial conditions and control parameters are the same in both scenarios, and all the state variables are assumed to be measurable. In both cases, the biomass is regulated and the exponential reference trajectory is tracked after $0.8 \mathrm{~h}$; the convergence time is identical for the two models, as can be seen in Figure 6. The offset due to initialization and model mismatch is forced to zero by the integral term in the GMC control formulation.

However, in the case of the controller based on the full-order model, simulation shows that the feed-rate reaches unrealistic high values as well as the substrate concentration, which gets close to $S_{i n}$. Indeed, as established in Section 3.1.1, for every set biomass value $X_{\text {set }}$, two possible substrate concentrations can be obtained from the model and only one corresponds to realistic growth conditions.

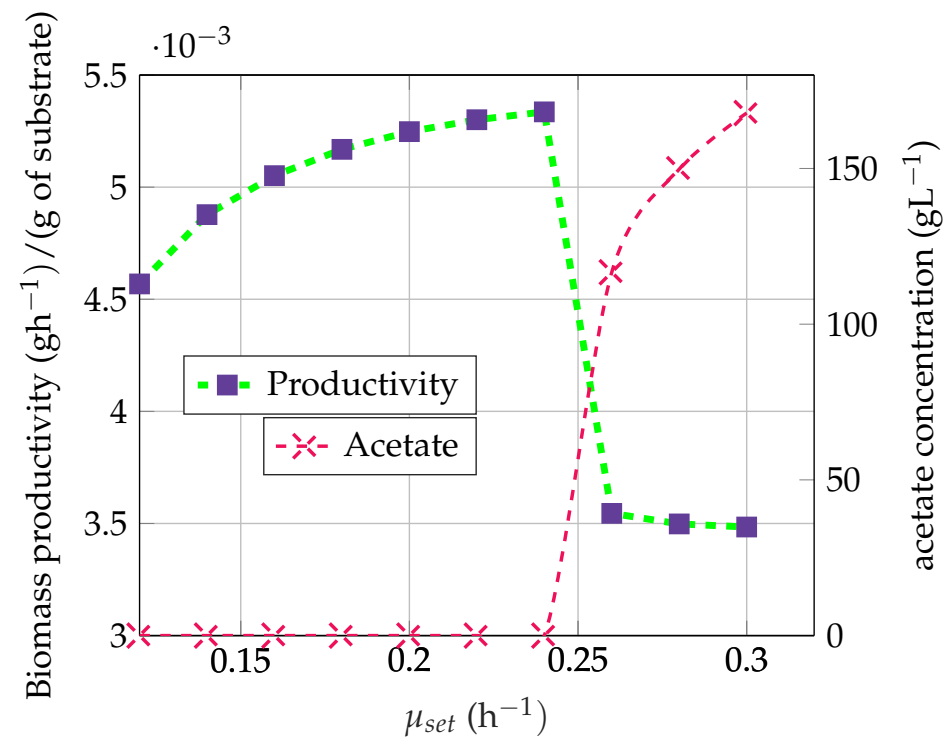

Figure 4. Biomass productivity (green) and acetate production (red) for different $\mu_{\text {set }}$ values.

Conversely, in the case of the control law derived from the reduced model formulation, the system converges to the desired trajectory corresponding to low substrate concentration. The substrate and the acetate concentrations are in the expected practical range, and the calculated flow rate is more realistic.

While the full-order system may indeed converge to both substrate solutions, the reduced model, established under the assumption of low dilution rates, i.e., $D \xi_{f}=0$ and therefore $F_{i n}$, will always converge to the expected realistic trajectory.

To test further the robustness of the proposed controller, a sequence of Monte Carlo simulations is achieved, with $15 \%$ relative uncertainty on the plant parameters following a normal distribution and with the same control setting as the previous simulations. Five hundred Monte Carlo (MC) simulations were performed, and the histogram of the parameter $\bar{k}_{11}$ during the MC runs is shown in Figure 8. The biomass, substrate, acetate, and flow rate time evolutions are presented in Figure 9.

In all the runs, the corresponding reference substrate concentration $S_{\text {set }}$ is reached and the acetate concentration is equal to zero at the end of the culture, ending in respiratory mode. However, parameter variations imply a distribution of the final biomass concentration. Nevertheless, the goal of reaching high biomass concentrations while keeping the culture in good operating conditions is achieved in all experiments.

The GMC scheme responses to disturbances and setpoint change are illustrated in Figures 10 and 11 respectively. The considered disturbance is a step of $0.3 \mathrm{gL}^{-1}$ in the measured biomass concentration by the turbidity probe, introduced at $\mathrm{t}=5 \mathrm{~h}$ and lasting until the end of the simulation, since experiments 
have shown that the addition of antifoam can result in a bias on the online biomass signal. The results show a good disturbance rejection and a fast tracking recovery. Compared to the set-point change, the same simulation is achieved with a trajectory change from $\mu$ set $=0.18 \mathrm{~h}^{-1}$ to $\mu$ set $=0.22 \mathrm{~h}^{-1}$ at $\mathrm{t}=3 \mathrm{~h}$, before returning to $\mu$ set $=0.18 \mathrm{~h}^{-1}$ at $\mathrm{t}=6 \mathrm{~h}$. Obviously, the controller is able to handle all of these disturbances, providing a fast and robust behavior.
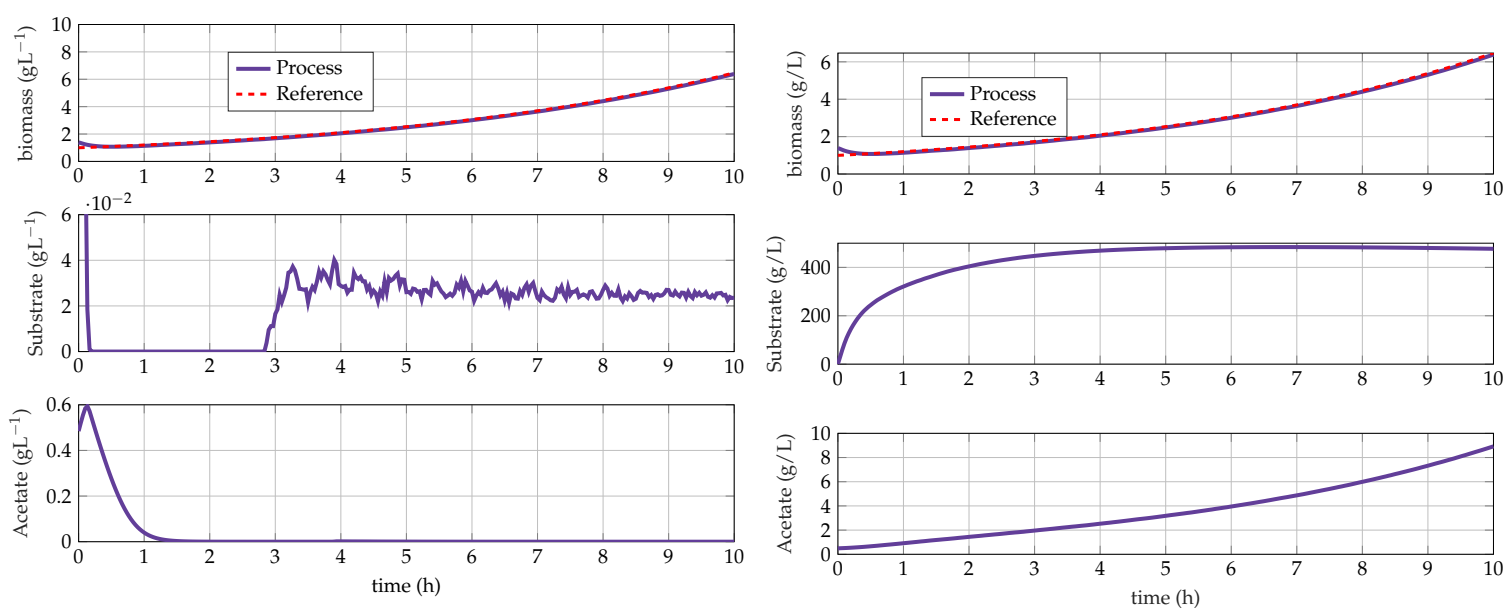

Figure 5. State variable evolutions with the full-order model (Right) and the reduced model (Left).
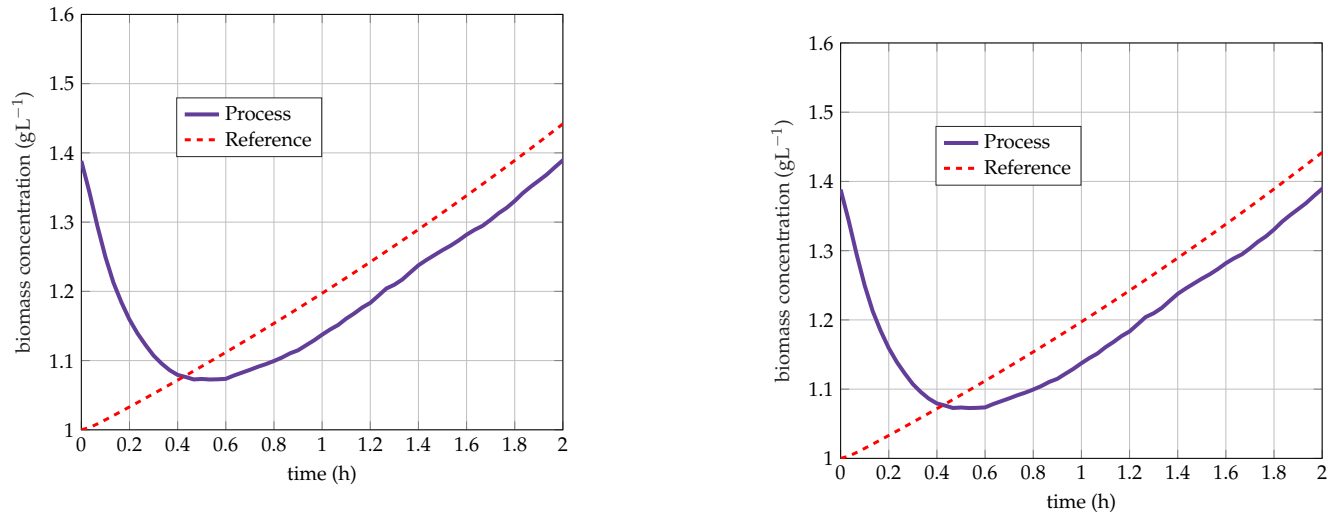

Figure 6. Biomass tracking with the full-order model (Right) and the reduced model (Left).
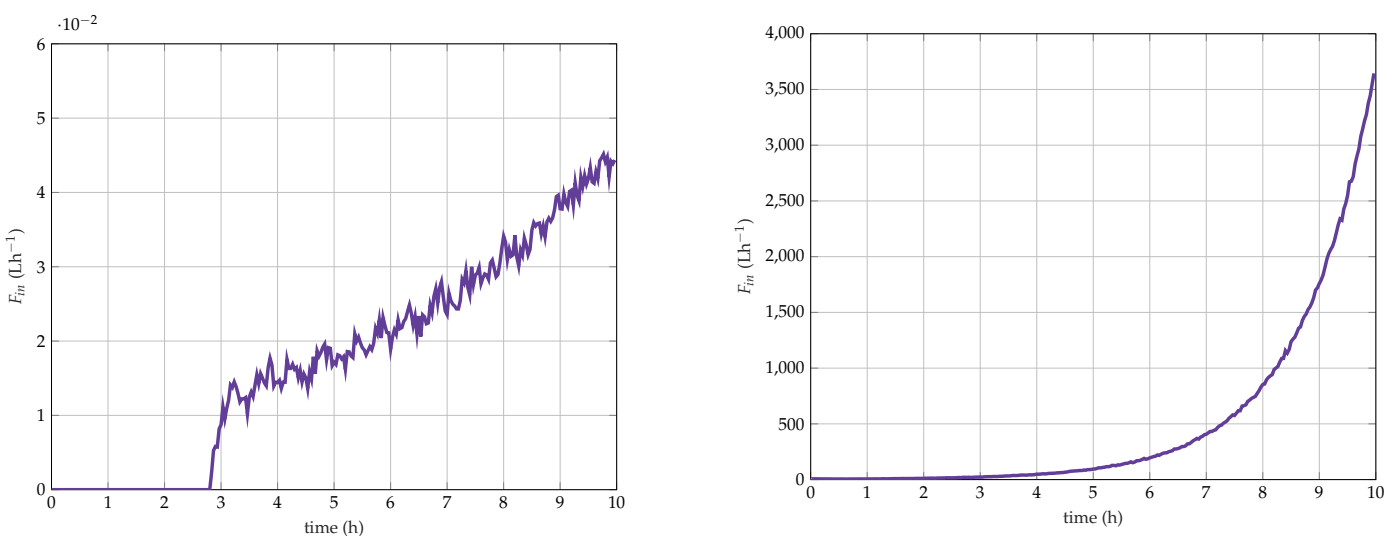

Figure 7. Flow rate evolution: full-order model (Right) and reduced model (Left). 


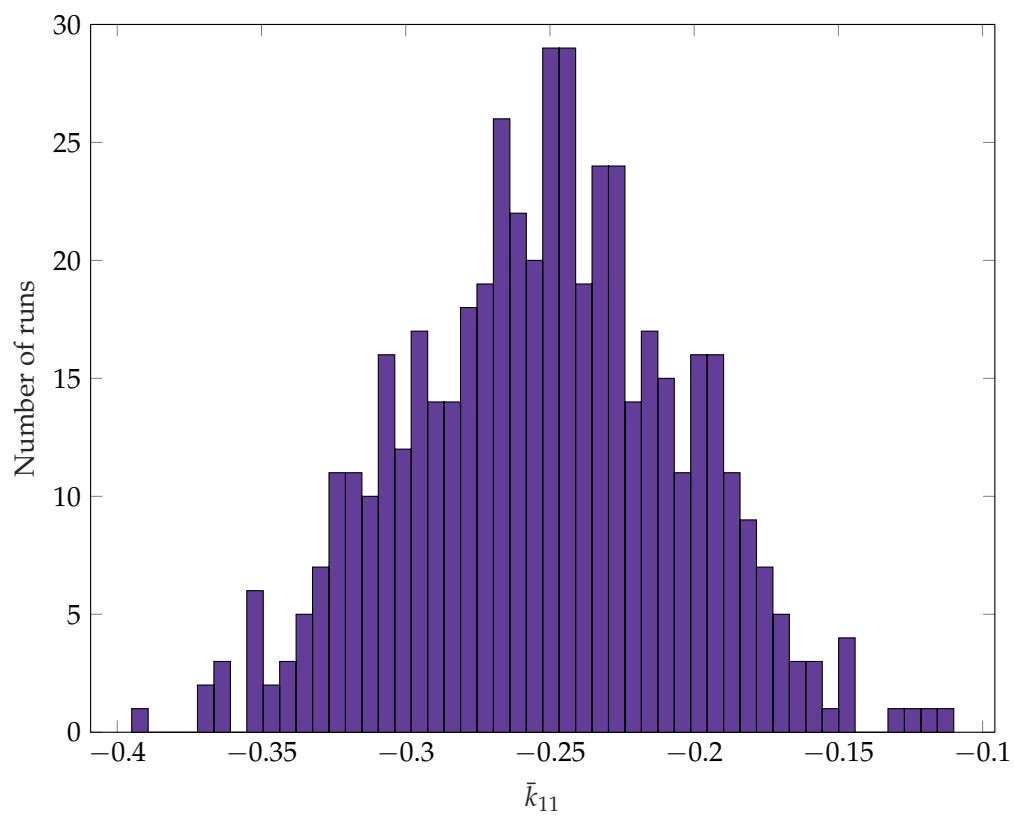

Figure 8. Histogram of the parameter $\bar{k}_{11}$ during 500 Monte Carlo runs.
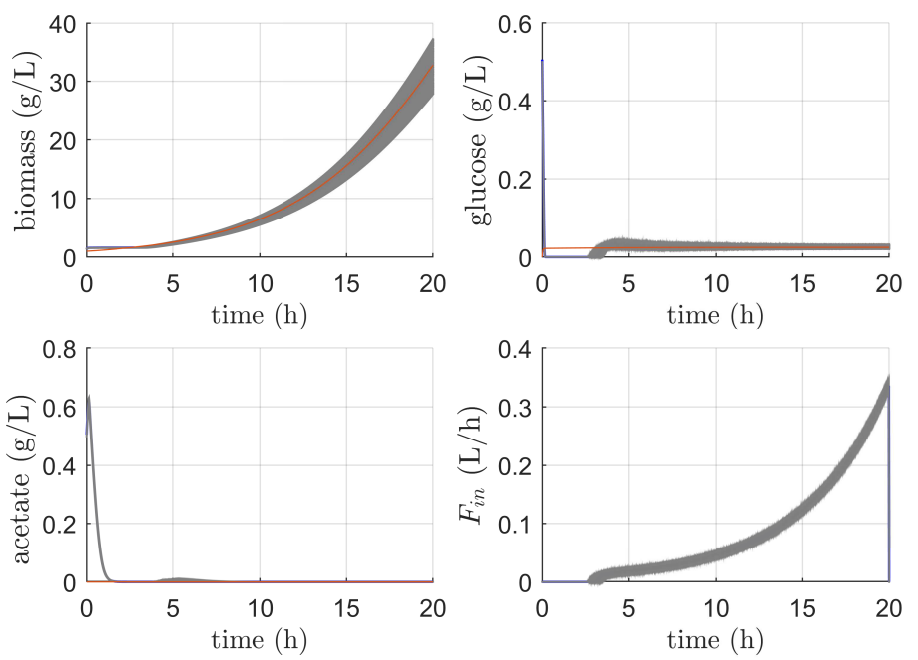

Figure 9. State variables and feed-rate evolution during 500 Monte Carlo runs.

In order to compare the performance of the proposed GMC strategy with other regular control schemes, a simulation is performed with a GMC controller, a first-order linearizing controller (FOC) [22,23], and a proportional-integral-derivative controller (PID). A reference profile is imposed with $\mu_{\text {set }}=0.18 \mathrm{~h}^{-1}$, and a step perturbation of $0.3 \mathrm{gL}^{-1}$ is added to the output signal. The first-order linearizing controller has the same structure as the GMC but without the integral term:

$$
\hat{F}=G_{1}\left(X_{r e f}-X\right)
$$

The PID controller has the following transfer function:

$$
C(s)=K_{p}+K_{i} \frac{1}{s}+K_{d} \frac{s}{1+T_{f} s}
$$



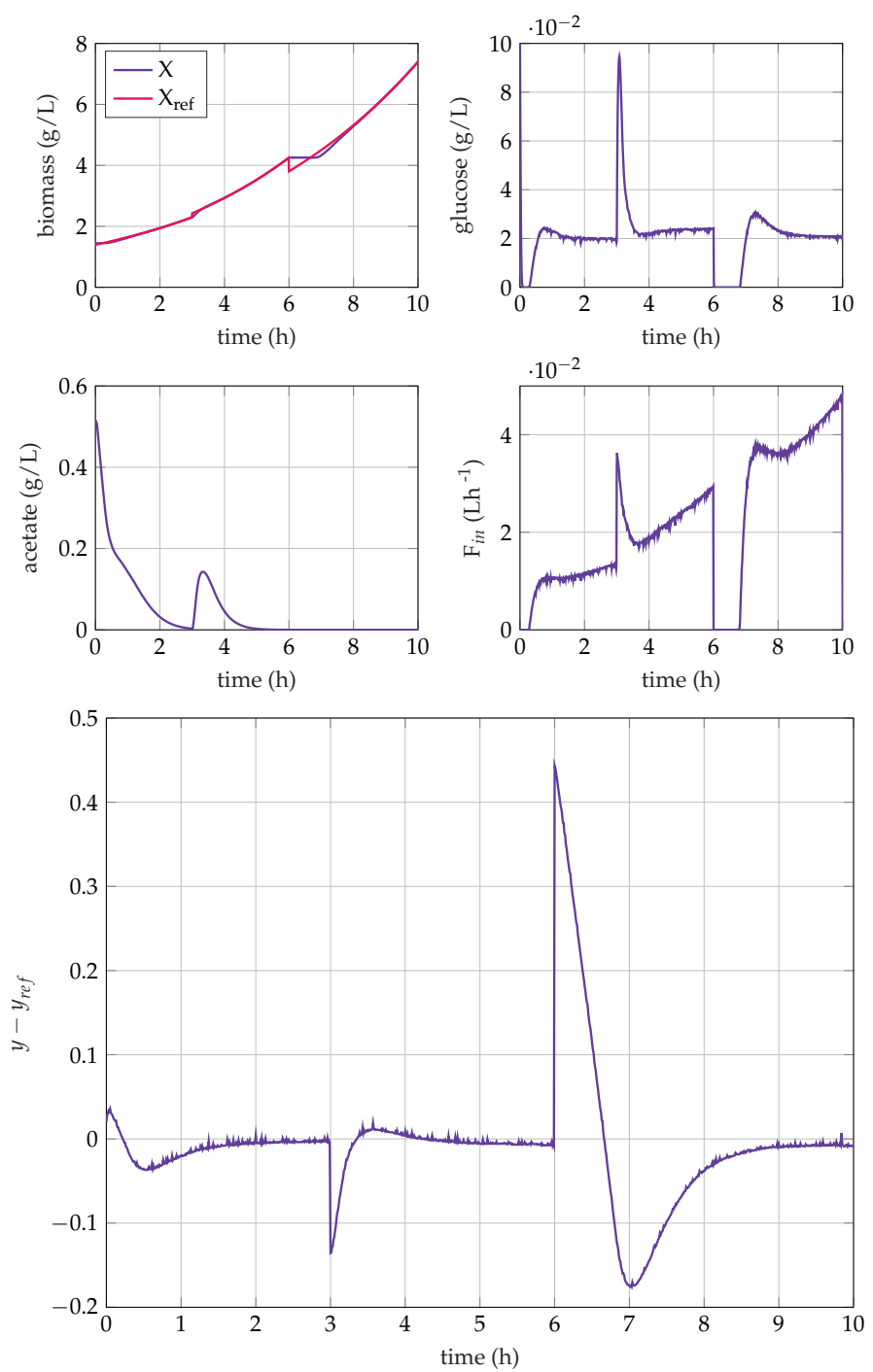

Figure 10. Closed-loop response to a setpoint change $\mu$ set $=0.18 \mathrm{~h}^{-1}$ and $\mu$ set $=0.22 \mathrm{~h}^{-1}$.

The PID parameters are chosen to have an overall dynamic behavior close to the one obtained by the GMC, the parameters values are $K_{p}=0.07, K_{i}=0.01, K_{d}=0.01$, and $T_{f}=0.5$. The first-order controller gain $G_{1}$ is chosen equal to the GMC proportional gain.

Figure 12 shows state variable $\left(X, S\right.$, and $A$ ) responses, the controller output $\left(F_{\text {in }}\right)$, and the biomass output tracking error $(y-y$ ref $)$ for the three controllers (GMC, FOC, and PID). As can be seen, the three controllers managed to track the biomass trajectory correctly, with slightly better convergence charasteristics for the GMC controller, until the introduction of the disturbance at $\mathrm{t}=$ $7 \mathrm{~h}$ which provokes different controller reactions. The GMC control scheme presents by far the best robust behavior. Furthermore, parameter tuning is straightforward in the GMC case compared to the PID controller due to the nonlinear nature of the process, which is imbedded in the design of the GMC.

It is noteworthy to point out that, in the case of the PID controller, a metabolic switch from the respirative to the respiro-fermentative modes occurred at $t=10.5 \mathrm{~h}$, leading to acetate formation due to substrate excess $\left(S>S_{\text {crit }}\right)$, and thus, the control output (feed-rate) strayed from its initial exponential curve. 

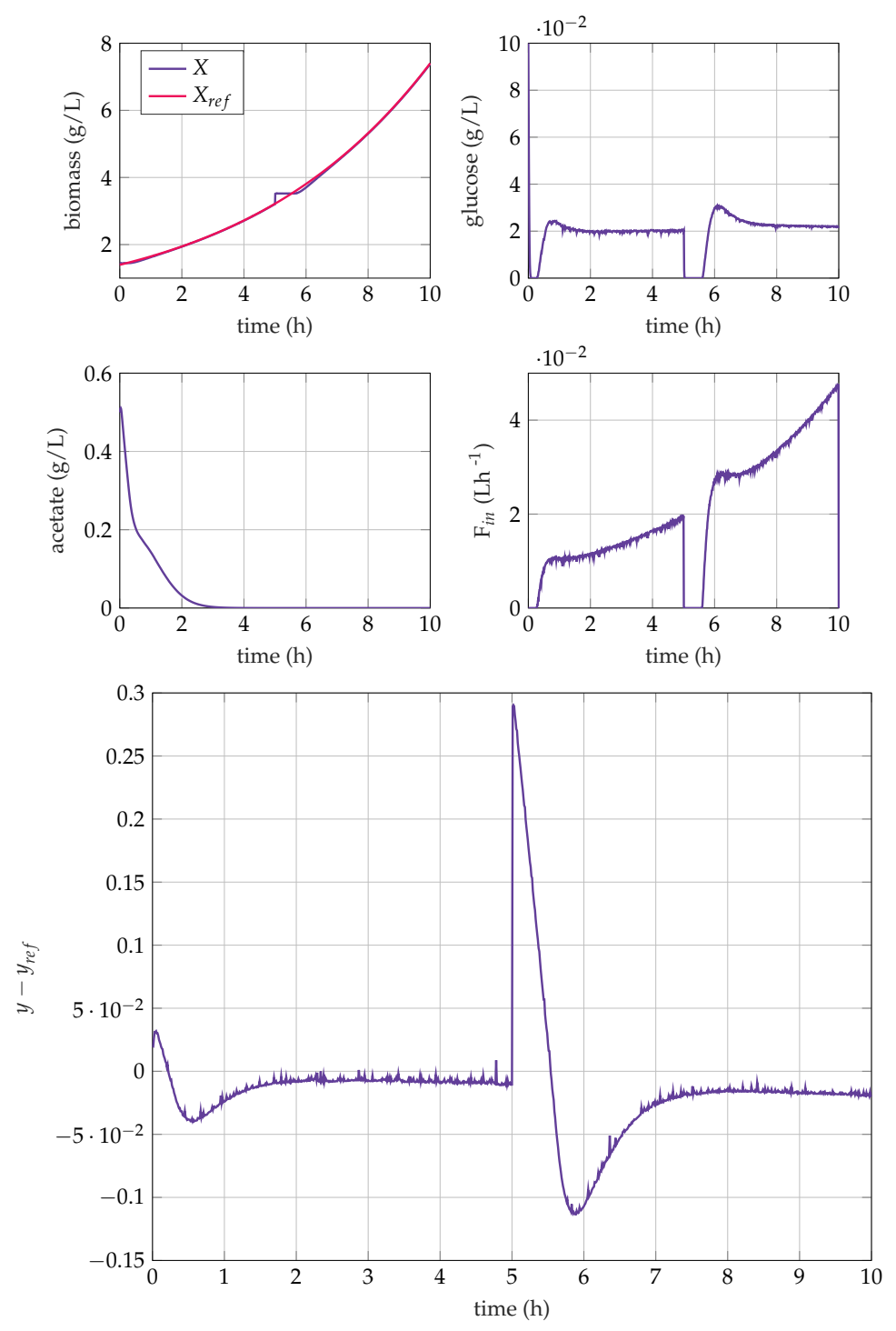

Figure 11. Closed-loop response to a disturbance on the biomass signal at $t=5 \mathrm{~h}$. 

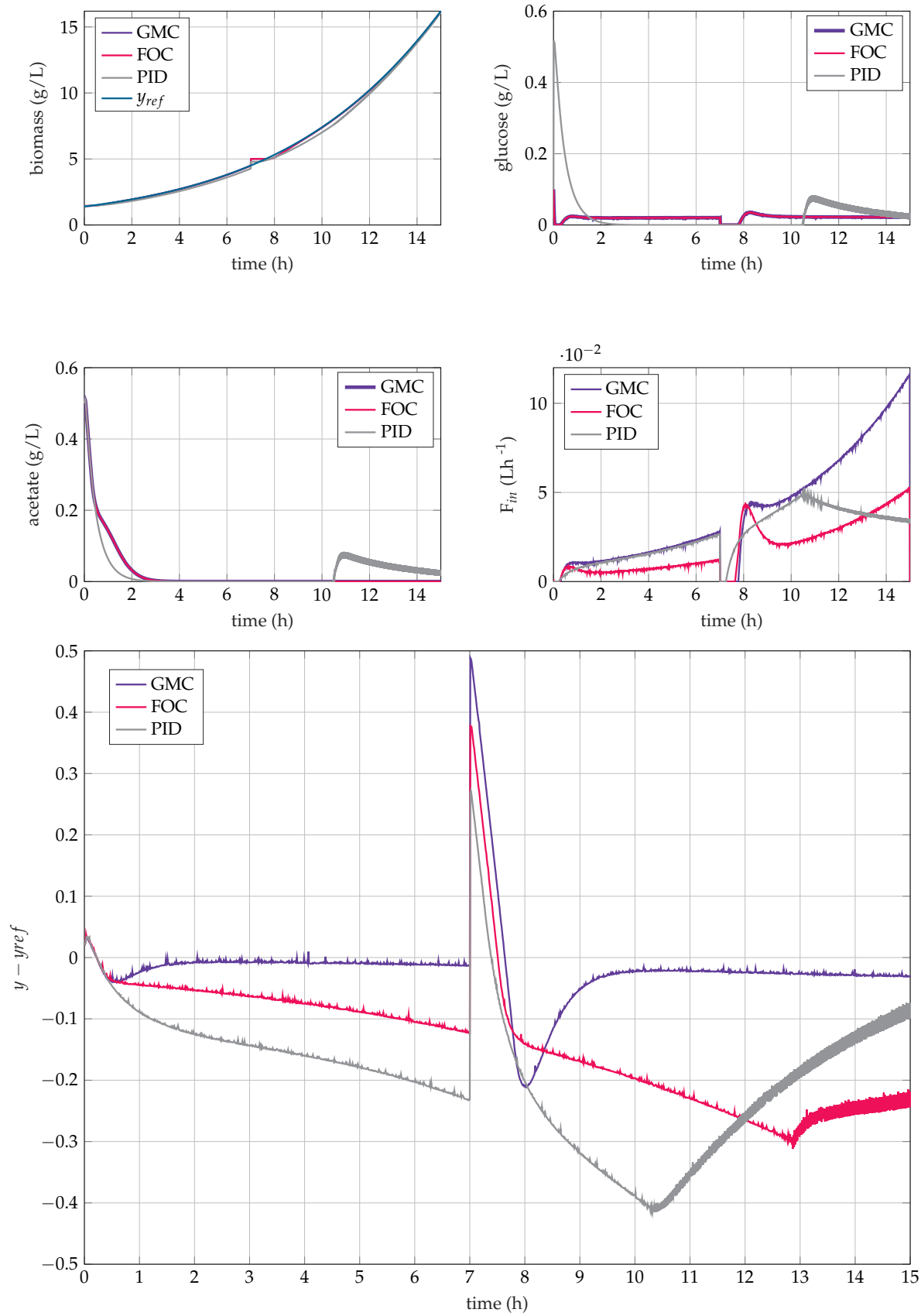

Figure 12. Comparison of the GMC performance with a first-order controller (FOC) and a PID controller.

\section{Materials and Methods}

\subsection{Microbial Strain}

The E. coli BL21 (DE3) strain was used in the bioreactor experiments. BL21 is known to lead to a low acetate formation compared to $E$. coli $\mathrm{K} 12$, which is suitable for high cell-density cultivation as well as a lower sensitivity to varying growth conditions [30]. 


\subsection{Growth Media and Culture Conditions}

The media used during the different stages of the cultures are the Lysogeny broth (LB) medium and a minimal growth medium (M9) $[23,26]$. Their respective compositions for the batch (precultures and bioreactor) and fed-batch cultures are given in Tables 7 and 8. During the preparation, solutions were filtered and sterilized in autoclave $\left(121^{\circ} \mathrm{C}\right.$ at $>15$ psi for 30 minutes) to avoid contamination.

Table 7. Composition of the Lysogeny broth (LB) medium.

\begin{tabular}{lc}
\hline Component & Concentration $\left(\mathrm{gL}^{-\mathbf{1}}\right)$ \\
\hline Peptone & 10 \\
yeast extract & 5 \\
$\mathrm{NaCl}$ & 6 \\
\hline
\end{tabular}

Table 8. Composition of the M9 medium.

\begin{tabular}{lcc}
\hline Component & Batch $\left(\mathbf{g L}^{-\mathbf{1}}\right)$ & Fed-Batch $\left(\mathbf{g L}^{-\mathbf{1}}\right)$ \\
\hline Glucose & $5-10$ & 500 \\
$\mathrm{Na}_{2} \mathrm{HPO}_{4}$ & 6 & - \\
$\mathrm{KH}_{2} \mathrm{PO}_{4}$ & 3 & - \\
$\mathrm{NH}_{4} \mathrm{Cl}$ & 1 & 10 \\
$\mathrm{NaCl}$ & 0.5 & - \\
$\mathrm{MgSO}_{4} \cdot 7 \mathrm{H}_{2} \mathrm{O}$ & 0.12 & 4 \\
\hline
\end{tabular}

The cryogenic culture was incubated for $24 \mathrm{~h}$ on LB-agar petri dishes at $37^{\circ} \mathrm{C}$. A single colony was transferred to $125 \mathrm{~mL}$ shaking flasks containing $20 \mathrm{~mL}$ LB-medium and incubated for $8 \mathrm{~h}$ on a shaker. A volume of $10 \mathrm{~mL}$ of this culture was then inoculated into $500 \mathrm{~mL}$ shake flasks containing $250 \mathrm{~mL}$ of the M9 media and incubated overnight $(14-16 \mathrm{~h})$ at $37^{\circ} \mathrm{C}$ and $200 \mathrm{RPM}$. This culture is used to innoculate the reactor and to start the batch phase.

\subsection{Reactor Setup}

The cultivations were performed in a bioreactor consisting of a $5 \mathrm{~L}$ jacketed glass vessel and a Digital Control Unit (DCU) (BIOSTAT B plus, Sartorius Stedim Biotech, Germany). The reactor is equipped with a water jacket and an agitation motor.

\subsection{Online Measurements}

The monitoring of the cultures is possible thanks to a potentiometric $\mathrm{pH}$ sensor (Hamilton, Switzerland), an optical dissolved oxygen $\left(\mathrm{pO}_{2}\right)$ probe (Hamilton, Switzerland), and a temperature sensor (Sartorius, Germany). In addition, biomass concentration is available online via an absorption-based photometric turbidity prepared probe (Fundalux II, Sartorius, Germany).

\subsection{Offline Measurements}

During the fermentation, samples were taken every hour. The biomass concentration was obtained by measuring the optical density at $600 \mathrm{~nm}\left(\mathrm{OD}_{600}\right)$ in a Shimadzu UV Spectrophotometer (Pharmacia Biotech, USA) correlated with dry cell weight (DCW) using a calibration curve. The conversion factor for $\mathrm{OD}_{600}$ to $\mathrm{DCW}$ was 0.39 . The supernatant was stored at $-8{ }^{\circ} \mathrm{C}$ for later analysis using enzymatic assay kits for acetate (Megazyme, Ireland), and the Dinitro Salicylic Acid (DNS) method was used for glucose. The sensitivity level of analytical methods is considered around $0.1 \mathrm{gL}^{-1}$. 


\subsection{Operating Conditions}

The batch and fed-batch fermentations were conducted under controlled conditions. The $\mathrm{pH}$ is regulated at 7 by adding solutions of $12.5 \%$ ammonium hydroxide (base) or phosphoric acid $0.5 \mathrm{M}$ (acid). Dissolved oxygen was ensured to be above $30 \%$ air saturation by manipulating in cascade the agitation speed and, when the maximum agitation is reached, the air flow. Minimum values for airflow and agitation were imposed ( $1 \mathrm{~L} \mathrm{~min}^{-1}$ and $200 \mathrm{rpm}$, respectively). The temperature is controlled by the DCU at $37^{\circ} \mathrm{C}$ using a heating water jacket.

\subsection{Cultures}

The cell cultures consist in a batch phase followed by a fed-batch operation. The preculture is prepared and incubated overnight as explained in Section 6.2. The initial $\mathrm{OD}_{600}$ in the reactor reaches 0.3-0.6. The batch fermentation had a starting volume of $3.5 \mathrm{~L}$. A lower volume was not possible, since the biomass probe would not be completely immerged.

The batch phase was monitored during the day. Once the glucose was nearly depleted, the fed-batch phase started, and the feeding solution was added with a rate determined by the controller and applied by a reglo-digital peristaltic pump (Ismatec, Germany).

\subsection{Algorithm Implementation}

The control and estimation algorithms were coded in Matlab for simulations and implemented online in LabView using shared library and MathScript nodes. The measurements provided by the DCU $\left(\mathrm{pH}, \mathrm{pO}_{2}\right.$, temperature, etc.) were monitored and saved by the MFCS software (Sartorius, Germany), and imported to LabView by shared libraries. The biomass signal was measured separately by a data acquisition device from national instruments (NI USB-6000USB Multifunction DAQ Device, National Instruments, USA) and squired in LabView using DAQMax library. Figure 13 shows the diagram of the different devices used for the online implementation.

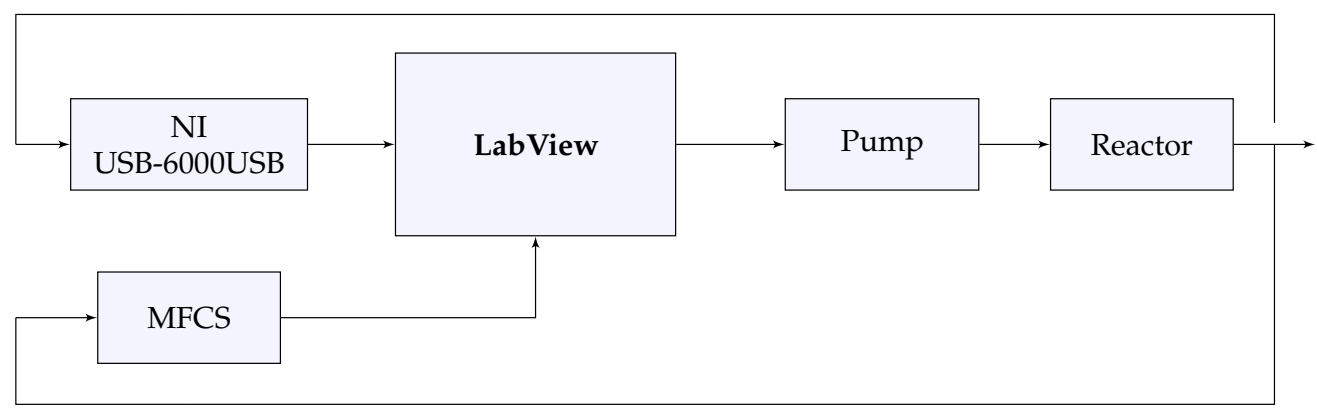

Figure 13. Real-time implementation diagram.

\section{Experimental Results and Discussion}

Two fed-batch experiments were performed to challenge the controller under real experimental conditions. The control and estimation parameters are given in Table 9. The control parameters $G_{1}$ and $G_{2}$ were tuned in simulation, the chosen response time $t_{r}$ is equal to $1 \mathrm{~h}\left(\omega_{0}=3 \mathrm{rad} / \mathrm{h}\right)$, and the damping ratio is fixed at $\xi=1$. As far as the parameter $\gamma$ estimation, the ramp model was used during the experiments.

Biomass, glucose, and acetate concentrations as well as the feed flow rate are shown in Figures 14 and 15. Operating conditions are also shown in Figures 16 and 17 and Tables 10 and 11. 
Table 9. Control and estimation parameters used in the experiments.

\begin{tabular}{|c|c|}
\hline Sampling Time & $T_{S}=0.05 \mathrm{~h}$ \\
\hline GMC parameters & $\begin{array}{c}G_{1}=6, G_{2}=9 \\
\xi=1, t_{r}=1 \mathrm{~h}, \omega_{0}=3 \mathrm{rad} / \mathrm{h}\end{array}$ \\
\hline Reference & $\begin{array}{c}F_{i n_{r e f}}=\frac{\mu_{s e t}}{k_{X S}} \frac{V_{0} X_{0} e^{\mu_{s e t}(t)}}{S_{\text {in }}} \\
\mu_{\text {set }}=0.18 \mathrm{~h}^{-1}, \quad k_{X S}=0.22\end{array}$ \\
\hline Estimator covariance matrices & $\begin{array}{c}P_{\text {Lin }}=\operatorname{diag}\left(\left[\begin{array}{lll}0.1 & 1 & 1\end{array}\right]\right) \\
Q_{\text {Lin }}=\operatorname{diag}\left(\left[\begin{array}{lll}0.01 & 0.1 & 0.1\end{array}\right]\right) \\
R_{\text {Lin }}=0.1\end{array}$ \\
\hline
\end{tabular}

During the fermentations, the initial biomass concentration ranged from $0.1-0.3 \mathrm{gL}^{-1}$ and reached $1.5-1.7 \mathrm{gL}^{-1}$ by the end of the batch phase, characterized by glucose depletion. The online flag for the end of the batch phase was the sudden increase of the $\mathrm{pH}$ and, consequently, the decrease of base addition, as can be seen in Figures 16 and 17. The fed-batch phase starts around 6-8 h of culture time, and the control algorithm is launched. During this period, the RPM increases due to the important glucose oxidation inducing an increasing cell demand for oxygen. The base is added to compensate the $\mathrm{pH}$ decrease.
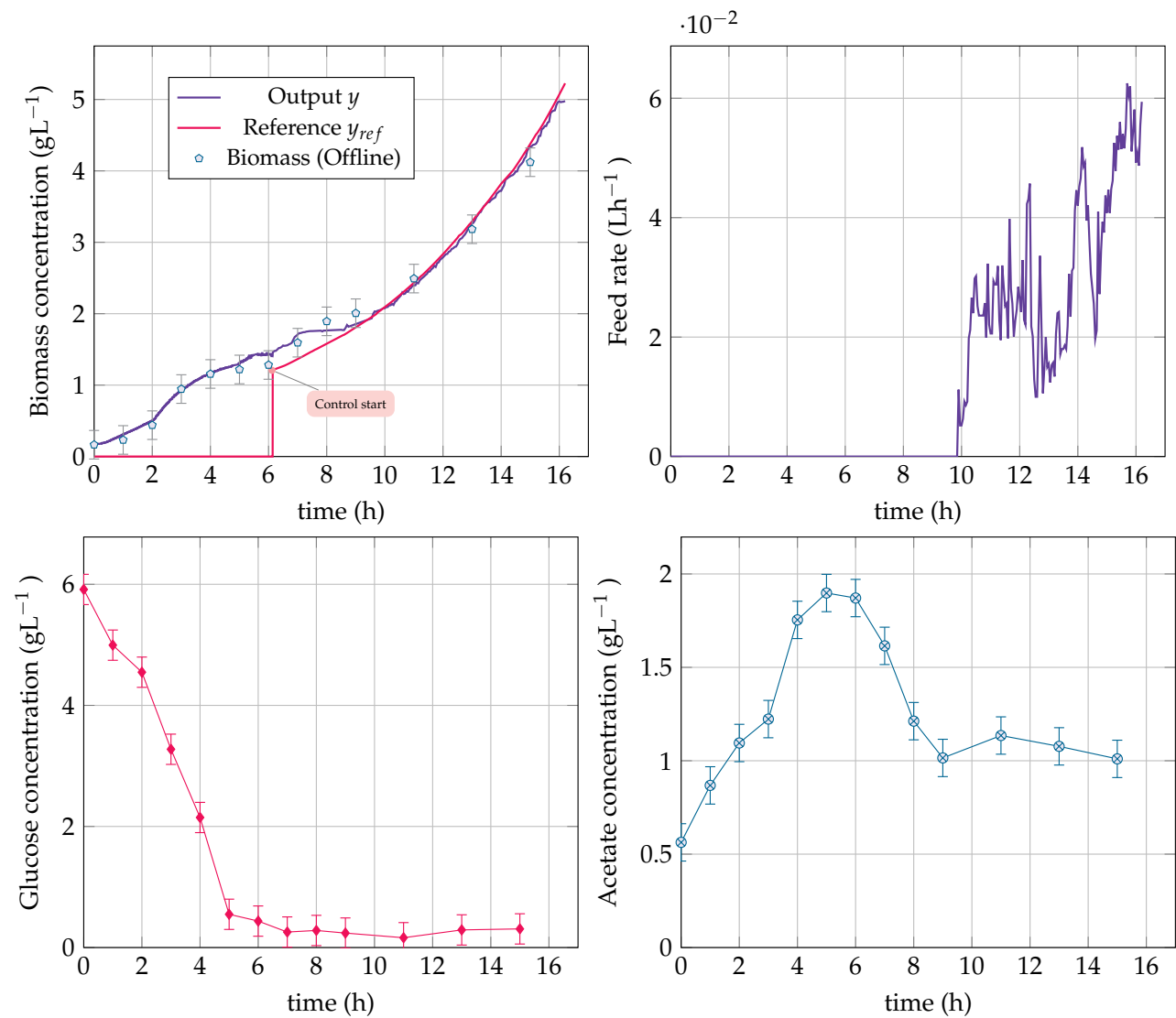

Figure 14. Experiment 1: Time evolution of the measured biomass, reference profile, glucose, acetate concentrations, and feed rate. 

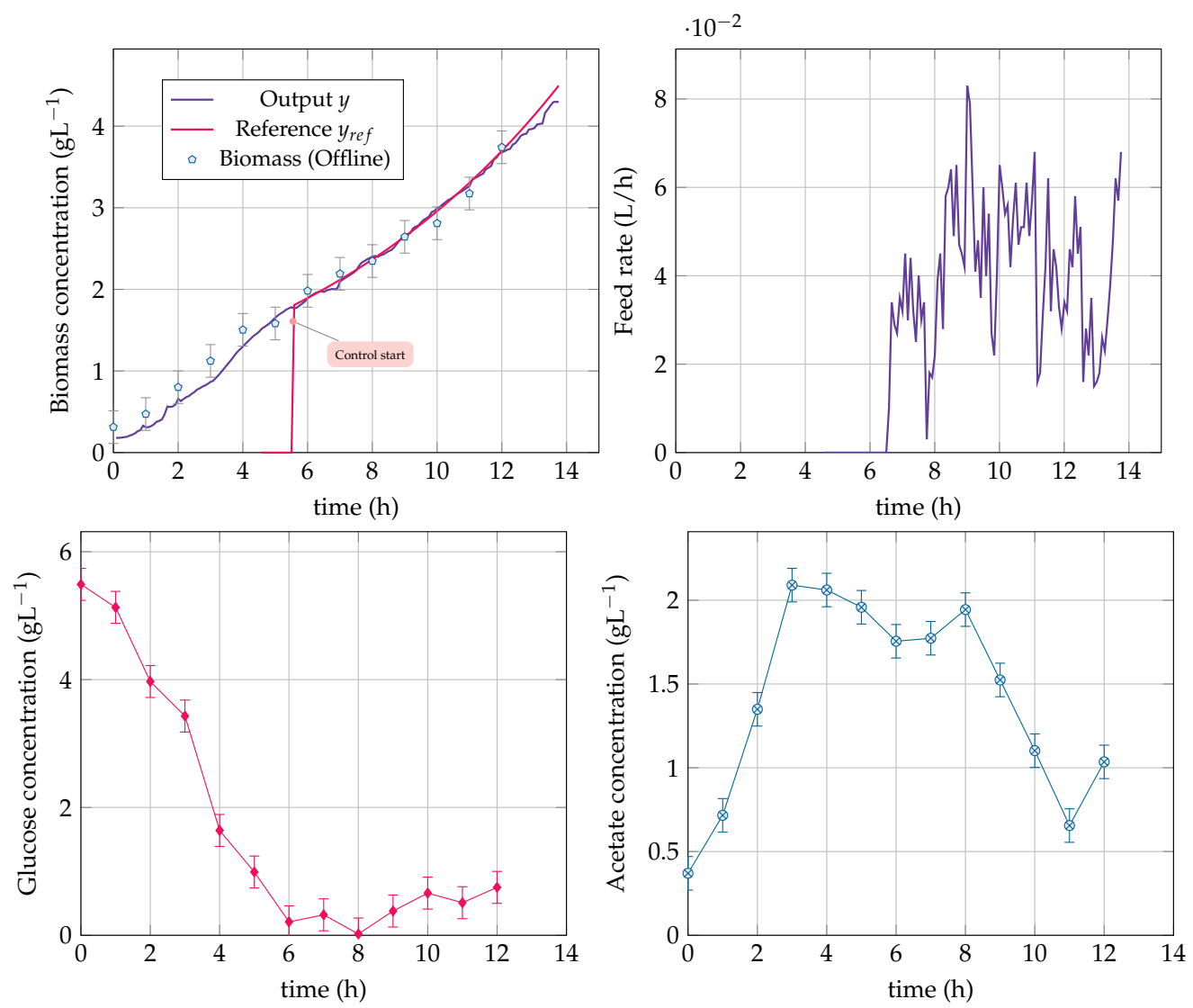

Figure 15. Experiment 2: Time evolution of the measured biomass, reference profile, glucose, acetate concentrations, and feed rate.

The imposed reference trajectory is $\mu_{\text {set }}=0.18 \mathrm{~h}^{-1}$, and the algorithm is launched with an initial biomass concentration lower than the measured one to avoid excessive feeding at the start of the fed-batch. In Figures 14 and 15, the biomass maintains an exponential growth close to the reference trajectory, showing that the regulation is efficient. The glucose concentration remains close to zero and almost constant during the fed-batch phase of both experiments, confirming the fast dynamics assumption presented in the model reduction section $(\dot{S}=0)$.

The acetate concentration remains under $2 \mathrm{gL}^{-1}$ during both experiments. The evolution indicates a metabolic switch between respirative and respiro-fermentative modes, as can be seen in Figure 14, where the acetate concentration increases from 0.5 to $1.7 \mathrm{gL}^{-1}$ due to overflow, and when the glucose is nearly depleted at $t=4 \mathrm{~h}$, the acetate concentration starts to decrease, i.e., the culture is in respirative mode. This behavior is reproduced during experiment 2 (Figure 15).

The generic model controller performance, in term of robustness, is quite satisfactory. The controller is able to maintain the biomass tracking error close to zero in both experiments despite the model mismatch resulting from modeling uncertainties and the use of basic minimal growth media (M9). The controller manages to adapt to the variations in the biomass signal by acting on the feed-flow rate. We can be seen in experiment 1 (Figure 14), the flow rate follows an exponential rate but is however heavily distorted due to the noisy estimation signal provided by the Kalman filter. We can see in Figure 18 that measurement noise indeed affects $\gamma$ estimation and, in turn, the calculated controller input. 
Table 10. Experiment 1: State variables and operating conditions during the culture.

\begin{tabular}{cccccc}
\hline $\begin{array}{c}\text { Age } \\
\mathbf{h}\end{array}$ & $\begin{array}{c}\text { Biomass } \\
\mathbf{g L}^{-\mathbf{1}}\end{array}$ & $\begin{array}{c}\text { Glucose } \\
\mathbf{g L}^{-\mathbf{1}}\end{array}$ & $\begin{array}{c}\text { Acetate } \\
\mathbf{g L}^{-\mathbf{1}}\end{array}$ & $\begin{array}{c}\mathbf{p H} \\
-\end{array}$ & $\begin{array}{c}\mathbf{p O}_{\mathbf{2}} \\
\mathbf{\%}\end{array}$ \\
\hline & & & & & \\
0 & 0.165 & 5.915 & 0.563 & 6.96 & 72 \\
1 & 0.232 & 4.995 & 0.868 & 6.95 & 56 \\
2 & 0.439 & 4.548 & 1.095 & 6.94 & 29.8 \\
3 & 0.945 & 3.275 & 1.223 & 6.96 & 29.3 \\
4 & 1.156 & 2.148 & 1.754 & 6.95 & 29.8 \\
5 & 1.219 & 0.549 & 1.898 & 6.94 & 31.4 \\
6 & 1.282 & 0.437 & 1.871 & 6.98 & 29.5 \\
7 & 1.594 & 0.256 & 1.615 & 7.02 & 30.4 \\
8 & 1.893 & 0.282 & 1.212 & 7.1 & 29.5 \\
9 & 2.007 & 0.239 & 1.015 & 7.06 & 28.4 \\
11 & 2.492 & 0.162 & 1.135 & 7.05 & 28.7 \\
13 & 3.183 & 0.291 & 1.077 & 6.94 & 29.9 \\
15 & 4.122 & 0.308 & 1.01 & 6.94 & 29.5 \\
\hline
\end{tabular}
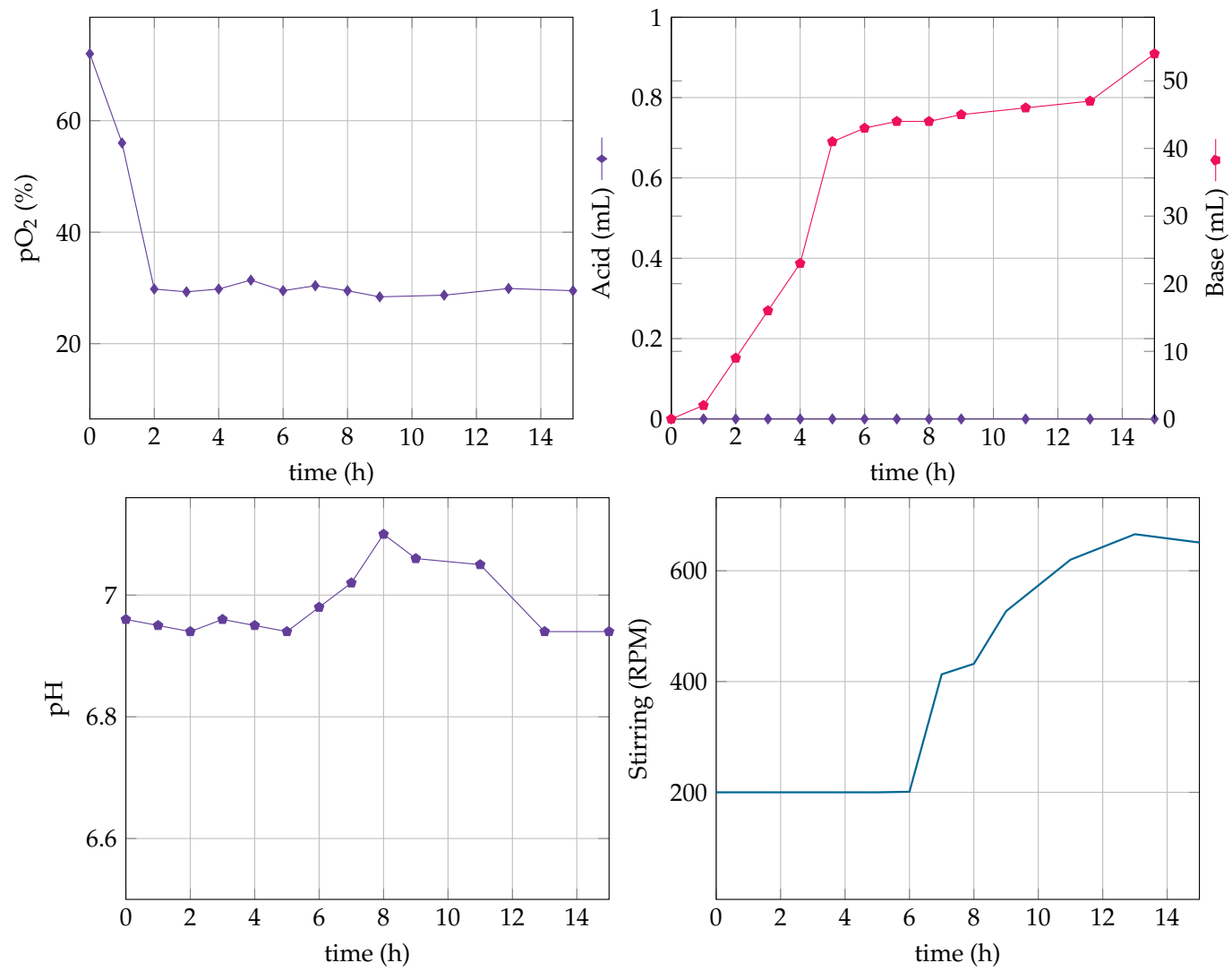

Figure 16. Experiment 1: Time evolution of $\mathrm{pO}_{2}$, acid and base concentrations, $\mathrm{pH}$, and stirring. 
Table 11. Experiment 2: State variables and operating conditions during the culture.

\begin{tabular}{cccccc}
\hline $\begin{array}{c}\text { Age } \\
\mathbf{h}\end{array}$ & $\begin{array}{c}\text { Biomass } \\
\mathbf{g L}^{-\mathbf{1}}\end{array}$ & $\begin{array}{c}\text { Glucose } \\
\mathbf{g L}^{-\mathbf{1}}\end{array}$ & $\begin{array}{c}\text { Acetate } \\
\mathbf{g L}^{-\mathbf{1}}\end{array}$ & $\begin{array}{c}\mathbf{p H} \\
-\end{array}$ & $\begin{array}{c}\mathbf{p O}_{\mathbf{2}} \\
\mathbf{\%}\end{array}$ \\
\hline & & & & & \\
0 & 0.312 & 5.49 & 0.370 & 7.1 & 71.2 \\
1 & 0.472 & 5.13 & 0.716 & 7.01 & 40.6 \\
2 & 0.801 & 3.97 & 1.349 & 6.93 & 26.1 \\
3 & 1.123 & 3.43 & 2.090 & 6.94 & 26.6 \\
4 & 1.504 & 1.64 & 2.061 & 6.95 & 30.5 \\
5 & 1.582 & 0.99 & 1.958 & 6.95 & 27.8 \\
6 & 1.982 & 0.21 & 1.755 & 6.94 & 26.4 \\
7 & 2.191 & 0.32 & 1.773 & 6.95 & 27.1 \\
8 & 2.347 & 0.02 & 1.944 & 6.95 & 26.9 \\
9 & 2.644 & 0.38 & 1.524 & 6.94 & 26.3 \\
10 & 2.809 & 0.66 & 1.102 & 6.95 & 28.6 \\
11 & 3.174 & 0.51 & 0.655 & 6.95 & 29.7 \\
12 & 3.741 & 0.75 & 1.035 & 6.94 & 28.9 \\
\hline
\end{tabular}
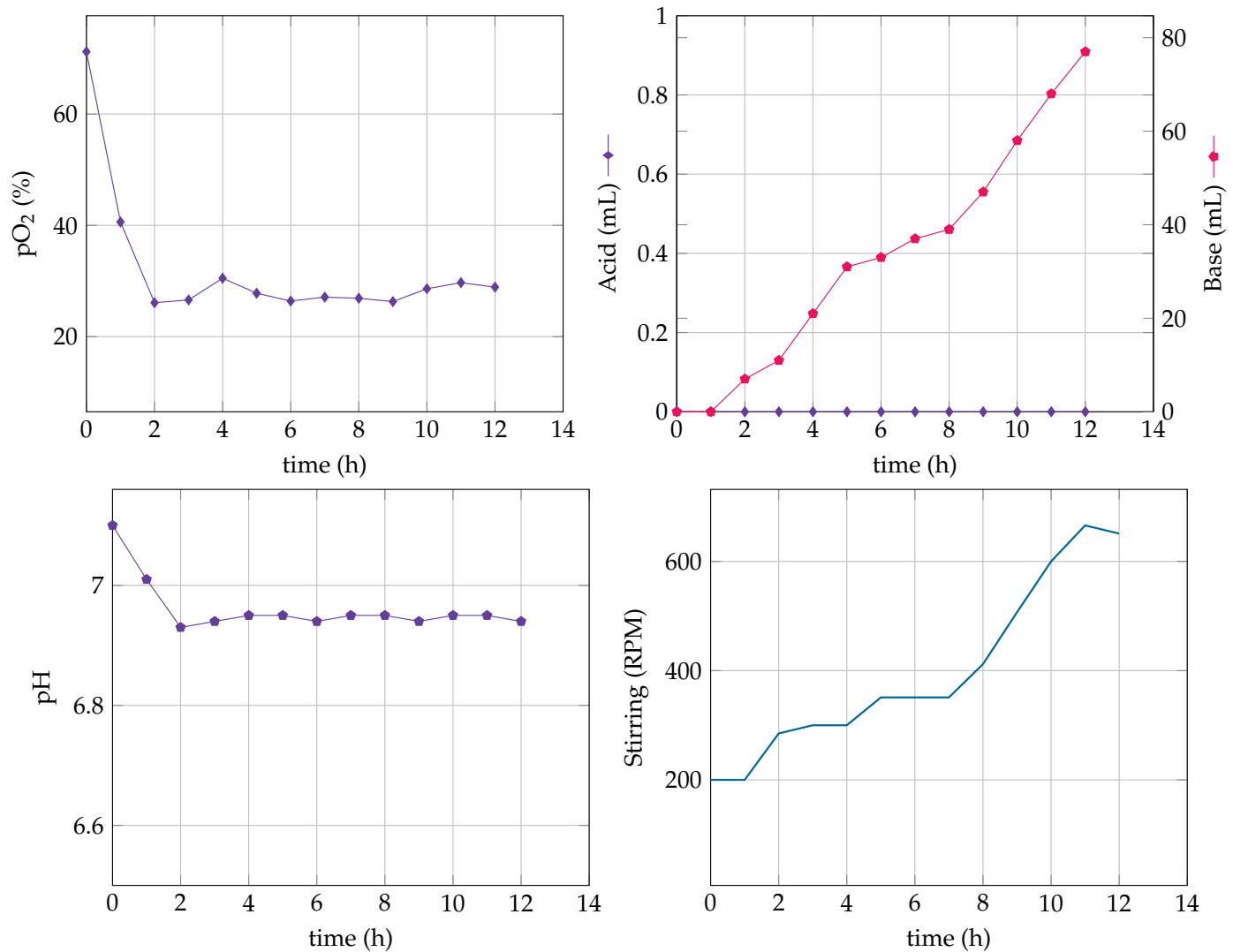

Figure 17. Experiment 2: Time evolution of $\mathrm{pO}_{2}$, acid and base concentrations, $\mathrm{pH}$, and stirring.

In order to compare the experimental results with the model predictions, a simulation using the nominal model is performed with the reference profile of the first experiment and under the same operating and control conditions (same parameter values). As shown in Figure 19, the ideal model control input $F_{\text {in }}$ follows the same tendency as the expertimental one. 
Moreover, the initial biomass trajectories are different, indicating a probable model/experiment parameter mismatch. The GMC controller is therefore able to track the right biomass concentration trajectory despite model uncertainties and measurement noise.
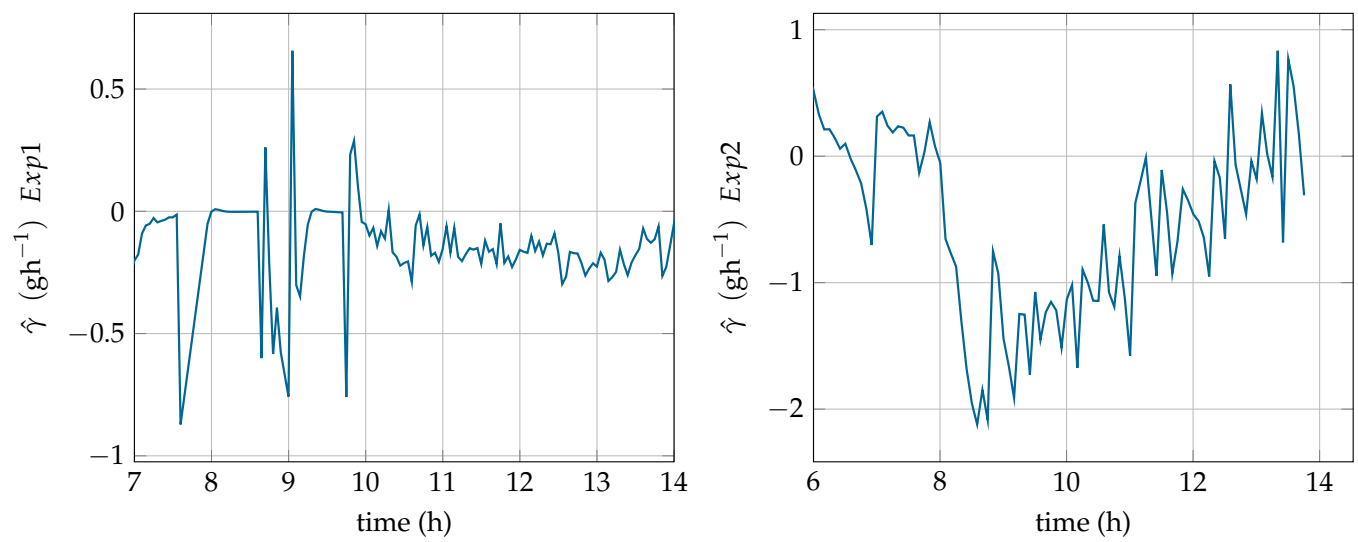

Figure 18. $\hat{\gamma}$ estimation during experiment 1 (left) and experiment 2 (right).
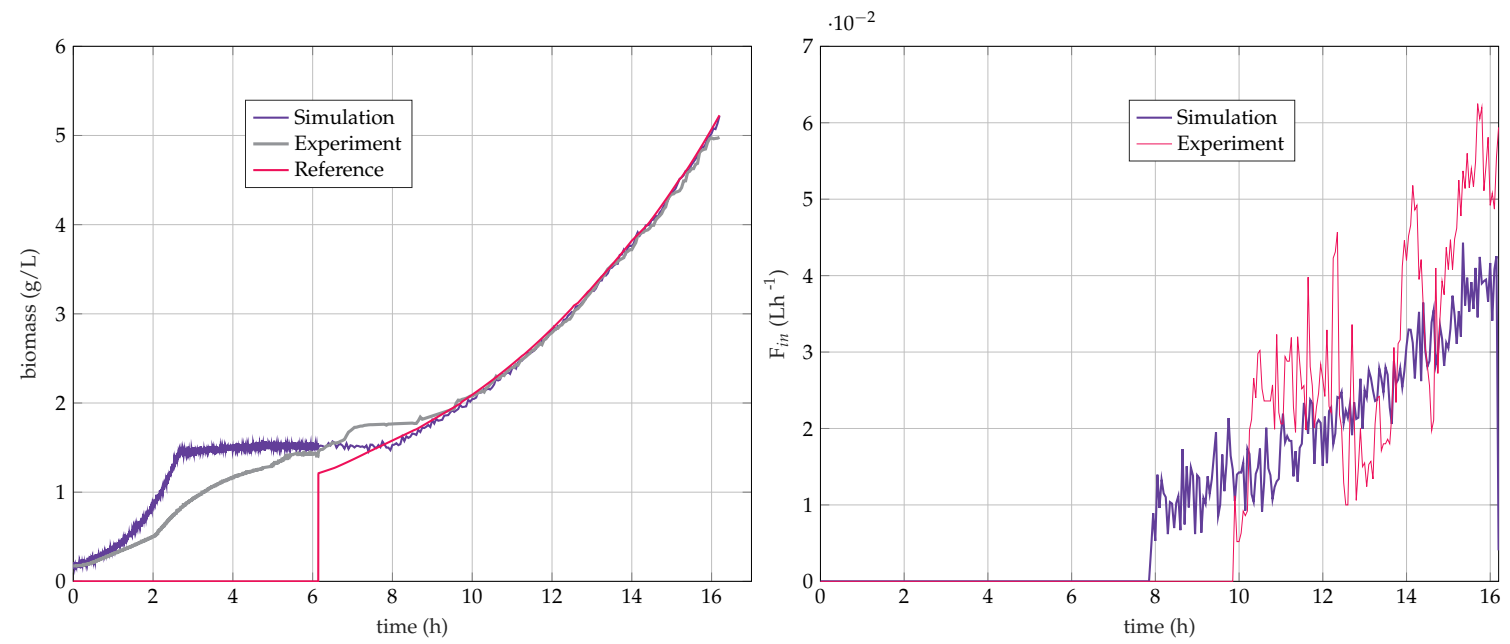

Figure 19. Experiment 1: Comparison between the simulated and experimental biomass response and the corresponding feeding profile $F_{i n}$.

\section{Conclusions}

The goal of this study is to develop a control strategy to regulate the biomass growth of E. coli BL21 (DE3) cultures in a fed-batch mode. The proposed strategy is generic model control, based on input-output linearization and a proportional-integral control law. Model reduction is applied to a mechanistic model describing the overflow metabolism. This model reduction allows obtaining a control law which is independent of the specific growth rates. GMC is combined to a parameter estimator provided by a linear Kalman filter.

Numerical simulations are carried out to test and tune the control and estimation algorithms. Results show that the accuracy and robustness of the proposed control strategy are quite satisfactory.

Fed-batch experiments of a BL21(DE3) E. coli strain are achieved with a lab-scale bioreactor, and results show that the biomass profile correctly tracks the given reference and that the controller manages to keep the culture in suitable growth conditions.

The combined GMC-Kalman strategy has the advantage of great simplicity of implementation and adaptability to the measurement environment. In the present setup, only online biomass measurements 
are used, but the availability of a gas analyzer could provide missing information on OTR and CTR, leading to a simplification of the control law.

Author Contributions: Conceptualization: S.T., D.D., A.V.W.; methodology: M.A., L.D., S.T.; computer implementation: M.A., experimental study: M.A, L.D.; writing-original draft preparation: M.A., L.D.; writing-review and editing: S.T., A.V.W.; supervision: A.V.W., S.T., D.D. All authors have read and agreed to the published version of the manuscript.

Funding: This research received no external funding.

Conflicts of Interest: The authors declare no conflict of interest.

\section{Appendix A}

Theoritical expression of the $\alpha_{j}$ coefficients presented in Equation (23), calculated using Matlab Symbolic Toolbox:

$$
\begin{aligned}
& \alpha_{1}=9 S_{i n} K_{O S}-9 K_{S} K_{O S}+50 K_{S} k_{X 1} q_{O_{\max }}-50 K_{S} k_{X 2} q_{O_{\max }}-50 S_{i n} k_{X 1} q_{O_{\max }} \\
& +50 S_{i n} k_{X 2} q_{O_{\max }}-50 S_{\text {in }} K_{O S} k_{X 2} q_{S_{\max }} \\
& \alpha_{2}=+50 K_{O S} q_{S_{\max }} \\
& \alpha_{3}=81 K_{S}^{2} K_{O S}^{2}-900 K_{S}^{2} K_{O S} k_{X 1} q_{O_{\max }}+900 K_{S}^{2} K_{O S} k_{X 2} q_{O_{\max }}+2500 K_{S}^{2} k_{X 1}^{2} q_{O_{\max }}^{2} \\
& -5000 K_{S}^{2} k_{X 1} k_{X 2} q_{O_{\max }}^{2}+2500 K_{S}^{2} k_{X 2}^{2} q_{O_{\max }}^{2}-900 K_{S} S_{i n} K_{O S}^{2} k_{X 2} q_{S_{\max }} \\
& +162 K_{S} S_{i n} K_{O S}^{2}+5000 K_{S} S_{i n} K_{O S} k_{X 1} k_{X 2} q_{O_{\max }} q_{S_{\max }}-1800 K_{S} S_{i n} K_{O S} k_{X 1} q_{O_{\max }} \\
& -5000 K_{S} S_{i n} K_{O S} k_{X 2}^{2} q_{O_{\max }} q_{S_{\max }}+1800 K_{S} S_{i n} K_{O S} k_{X 2} q_{O_{\max }}+5000 K_{S} S_{i n} k_{X 1}^{2} q_{O_{\max }}^{2} \\
& -10000 K_{S} S_{i n} k_{X 1} k_{X 2} q_{O_{\max }}^{2}+5000 K_{S} S_{i n} k_{X 2}^{2} q_{O_{\max }}^{2}-900 K_{S} X \operatorname{set} K_{O S}^{2} q_{S_{\max }} \\
& +5000 K_{S} X s e t K_{O S} k_{X 1} q_{O_{\max }} q_{S_{\max }}-5000 K_{S} X s e t K_{O S} k_{X 2} q_{O_{\max }} q_{S_{\max }}+2500 S_{i n}^{2} K_{O S}^{2} k_{X 2}^{2} q_{S_{\max }}^{2} \\
& -900 S_{i n}^{2} K_{O S}^{2} k_{X 2} q_{S_{\max }}+81 S_{i n}^{2} K_{O S}^{2}+5000 S_{\text {in }}^{2} K_{O S} k_{X 1} k_{X 2} q_{O_{\max }} q_{S_{\max }}-900 S_{i n}^{2} K_{O S} k_{X 1} q_{O_{\max }} \\
& -5000 S_{i n}^{2} K_{O S} k_{X 2}^{2} q_{O_{\max }} q_{S_{\max }}+900 S_{i n}^{2} K_{O S} k_{X 2} q_{O_{\max }}+2500 S_{i n}^{2} k_{X 1}^{2} q_{O_{\max }}^{2} \\
& -5000 S_{i n}^{2} k_{X 1} k_{X 2} q_{O_{\max }}^{2}+2500 S_{i n}^{2} k_{X 2}^{2} q_{O_{\max }}^{2} \\
& \alpha_{4}=5000 S_{i n} K_{O S}^{2} k_{X 2} q_{S_{\max }}^{2}+900 S_{\text {in }} K_{O S}^{2} q_{S_{\max }}-5000 S_{i n} K_{O S} k_{X 1} q_{O_{\max }} q_{S_{\max }}+5000 S_{i n} K_{O S} k_{X 2} q_{O_{\max }} q_{S_{\max }} \\
& \alpha_{5}=2500 K_{O S}^{2} q_{S_{\max }}^{2} \\
& \alpha_{6}=2\left(9 K_{O S}-50 k_{X 1} q_{O_{\max }}+50 k_{X 2} q_{O_{\max }}-50 K_{O S} k_{X 2} q_{S_{\max }}\right)
\end{aligned}
$$

\section{Appendix B}
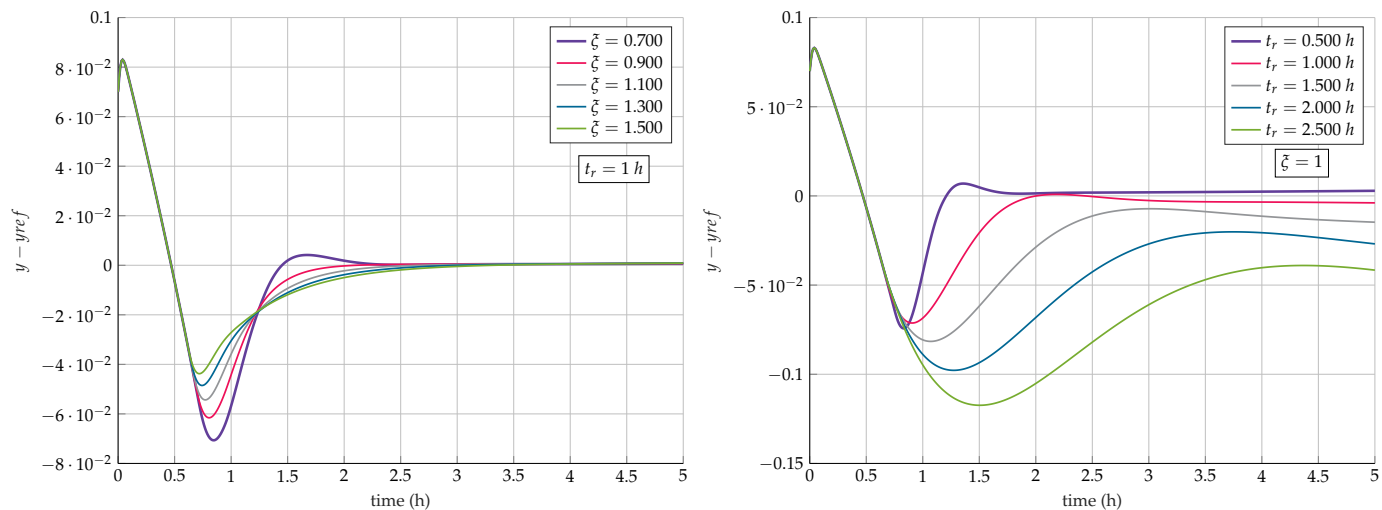

Figure A1. GMC biomass tracking response specification with parameters $\xi$ and $t_{r}$ for the $E$. coli BL21 model. 


\section{References}

1. Pontrelli, S.; Chiu, T.Y.; Lan, E.I.; Chen, F.Y.H.; Chang, P.; Liao, J.C. Escherichia coli as a host for metabolic engineering. Metab. Eng. 2018, 50, 16-46. [CrossRef] [PubMed]

2. Lee, S.Y. High cell-density culture of Escherichia coli. Trends Biotechnol. 1996, 14, 98-105. [CrossRef]

3. Han, K.; Lim, H.C.; Hong, J. Acetic acid formation in Escherichia coli fermentation. Biotechnol. Bioeng. 1992, 39, 663-671. [CrossRef]

4. Van De Walle, M.; Shiloach, J. Proposed mechanism of acetate accumulation in two recombinant Escherichia coli strains during high density fermentation. Biotechnol. Bioeng. 1998. [CrossRef]

5. $\quad$ Riesenberg, D.; Schulz, V.; Knorre, W.; Pohl, H.D.; Korz, D.; Sanders, E.; Roß, A.; Deckwer, W.D. High cell density cultivation of Escherichia coli at controlled specific growth rate. J. Biotechnol. 1991, 20, 17-27. [CrossRef]

6. Rothen, S.A.; Sauer, M.; Sonnleitner, B.; Witholt, B. Growth characteristics of Escherichia coil HB101[pGEc47] on defined medium. Biotechnol. Bioeng. 1998. [CrossRef]

7. Jana, S.; Deb, J.K. Strategies for efficient production of heterologous proteins in Escherichia coli. Appl. Microbiol. Biotechnol. 2005. [CrossRef]

8. Dewasme, L.; Coutinho, D.; Vande Wouwer, A. Adaptive and Robust Linearizing Control Strategies for Fed-Batch Cultures of Microorganisms Exhibiting Overflow Metabolism; Lecture Notes in Electrical Engineering; Springer: Berlin/Heidelberg, Germany, 2011; Volume 89, pp. 283-305. [CrossRef]

9. Dewasme, L.; Srinivasan, B.; Perrier, M.; Vande Wouwer, A. Extremum-seeking algorithm design for fed-batch cultures of microorganisms with overflow metabolism. J. Process Control 2011, 21, 1092-1104. [CrossRef]

10. Santos, L.; Dewasme, L.; Coutinho, D.; Vande Wouwer, A. Nonlinear model predictive control of fed-batch cultures of micro-organisms exhibiting overflow metabolism: Assessment and robustness. Comput. Chem. Eng. 2012, 39, 143-151. [CrossRef]

11. Gonzalez, K.; Tebbani, S.; Lopes, F.; Thorigné, A.; Givry, S.; Dumur, D.; Pareau, D. Regulation of lactic acid concentration in its bioproduction from wheat flour. Control Eng. Pract. 2016, 54, 202-213. [CrossRef]

12. Benattia, S.; Tebbani, S.; Dumur, D. Hierarchical Control Strategy based on Robust MPC and Integral Sliding mode-Application to a Continuous Photobioreactor. IFAC-PapersOnLine 2015, 48, 212-217. [CrossRef]

13. Dewasme, L.; Richelle, A.; Dehottay, P.; Georges, P.; Remy, M.; Bogaerts, P.; Vande Wouwer, A. Linear robust control of S. cerevisiae fed-batch cultures at different scales. Biochem. Eng. J. 2010, 53, 26-37. [CrossRef]

14. Rocha, I.; Ferreira, E. Model-based Adaptive Control of Acetate Concentration during the Production of Recombinant Proteins With E. Coli. IFAC Proc. Vol. 2002, 35, 461-466. [CrossRef]

15. Hafidi, G.; Tebbani, S.; Dumur, D.; Vande Wouwer, A. Nonlinear Model Predictive Control applied to E. coli Cultures. IFAC Proc. Vol. 2008, 41, 14570-14575. [CrossRef]

16. Renard, F.; Vande Wouwer, A.; Valentinotti, S.; Dumur, D. A practical robust control scheme for yeast fed-batch cultures-An experimental validation. J. Process Control 2006, 16, 855-864. [CrossRef]

17. Lee, P.; Sullivan, G. Generic model control (GMC). Comput. Chem. Eng. 1988, 12, 573-580. [CrossRef]

18. DeLisa, M.; Chae, H.; Weigand, W.; Valdes, J.; Rao, G.; Bentley, W. Generic model control of induced protein expression in high cell density cultivation of Escherichia coli using on-line GFP-fusion monitoring. Bioprocess Biosyst. Eng. 2001, 24, 83-91.

19. Costello, D.J.; Lee, P.L.; Greenfield, P.F. Control of anaerobic digesters using Generic Model Control. Bioprocess Eng. 1989. [CrossRef]

20. Douglas, P.L.; Fountain, P.S.; Sullivan, G.R.; Zhou, W. Model based control of a high purity distillation column. Can. J. Chem. Eng. 1994. [CrossRef]

21. Jenzsch, M.; Simutis, R.; Luebbert, A. Generic model control of the specific growth rate in recombinant Escherichia coli cultivations. J. Biotechnol. 2006, 122, 483-493. [CrossRef]

22. Bastin, G.; Dochain, D. On-line estimation and adaptive control of bioreactors. Anal. Chim. Acta 1991. [CrossRef]

23. Rocha, I. Model-Based Strategies for Computer-Aided Operation of Recombinant E. coli Fermentation; Universidade do Minho: Braga, Portugal, 2003.

24. Retamal, C.; Dewasme, L.; Hantson, A.L.; Vande Wouwer, A. Parameter estimation of a dynamic model of Escherichia coli fed-batch cultures. Biochem. Eng. J. 2018, 135, 22-35. [CrossRef] 
25. Sonnleitner, B.; Käppeli, O. Growth of Saccharomyces cerevisiae is controlled by its limited respiratory capacity: Formulation and verification of a hypothesis. Biotechnol. Bioeng. 1986, 28, 927-937. [CrossRef] [PubMed]

26. Dewasme, L.; Goffaux, G.; Hantson, A.L.; Vande Wouwer, A. Experimental validation of an Extended Kalman Filter estimating acetate concentration in E. coli cultures. J. Process. Control 2013, 23, 148-157. [CrossRef]

27. Peter, E.; Lee, L. Nonlinear Process Control: Applications of Generic Model Control; Springer: London, UK, 1993.

28. Zhou, W.; Lee, P.L.; Sullivan, G.R. Robust stability analysis of Generic Model Control. Chem. Eng. Commun. 1992, 117, 41-72. [CrossRef]

29. Welch, G.; Bishop, G. An Introduction to the Kalman Filter; University of North Carolina at Chapel Hill: Chapel Hill, NC, USA, 1995.

30. Müller, M.; Meusel, W.; Husemann, U.; Greller, G.; Kraume, M. Application of heat compensation calorimetry to an E. coli fed-batch process. J. Biotechnol. 2018, 266, 133-143. [CrossRef]

(C) 2020 by the authors. Licensee MDPI, Basel, Switzerland. This article is an open access article distributed under the terms and conditions of the Creative Commons Attribution (CC BY) license (http:/ / creativecommons.org/licenses/by/4.0/). 